J. DIFFERENTIAL GEOMETRY

96 (2014) 95-140

\title{
THE SKEIN ALGEBRA OF ARCS AND LINKS AND THE DECORATED TEICHMÜLLER SPACE
}

\author{
JULIEN ROGER \& TIAN YANG
}

\begin{abstract}
We define an associative $\mathbb{C}[[h]]$-algebra $\mathcal{A S}_{h}(\Sigma)$ generated by regular isotopy classes of arcs and links over a punctured surface $\Sigma$ which is a deformation quantization of the Poisson algebra $\mathcal{C}(\Sigma)$ of arcs and loops on $\Sigma$ endowed with a generalization of the Goldman bracket. We then construct a Poisson algebra homomorphism from $\mathcal{C}(\Sigma)$ to the algebra of smooth functions on the decorated Teichmüller space endowed with a natural extension of the Weil-Petersson Poisson structure described by Mondello. The construction relies on a collection of geodesic lengths identities in hyperbolic geometry which generalize Penner's Ptolemy relation, the trace identities and Wolpert's cosine formula. As a consequence, we derive an explicit formula for the geodesic lengths functions in terms of the edge lengths of an ideally triangulated decorated hyperbolic surface.
\end{abstract}

\section{Introduction}

The skein module $\mathcal{S}_{q}(M)$ of a 3-manifold $M$ was introduced independently by Turaev [Tu88] and Przytycki [Pr91] as a generalization of the Jones polynomial of a link in $S^{3}$, using as a key ingredient the Kauffman bracket skein relation. If the 3 -manifold is the product $\Sigma \times[0,1]$ of a surface $\Sigma$ by an interval, its skein module has a natural structure of an algebra, and is at the heart of the combinatorial approach to constructing a TQFT developed in [BHMV95]. This construction in turn has had many applications in low dimensional topology (see [FWW02] for example) and has been shown recently to be equivalent to the geometric approach to TQFT coming from conformal field theory (see the recent work of Andersen and Ueno [AU11]).

On the other hand, the skein algebra turns out to be deeply related to the $S L_{2}$-geometry of the underlying surface. More precisely, following the work of Turaev [Tu91], Bullock, Frohman and Kania-Bartoszyńska [Bu97, BFK99] and Przytycki and Sikora [PS00], the skein algebra can be understood as a deformation quantization of the $S L_{2}(\mathbb{C})$-character variety of $\Sigma$.

The first author was partially supported by the National Science Foundation grant DMS1207832 and the second author by a National Science Foundation research fellowship.

Received 10/10/2012. 
The goal of the present paper is the following: First, we extend the notion of skein algebra for a punctured surface by allowing for arcs between the punctures. In order to show that this construction is well-defined we revisit arguments found in [Go86, Pr91] and extend them when necessary. Second, following the approach of [BFK99], we relate our construction to geometric structures of $\Sigma$. In our approach, the relevant object turns out to be the decorated Teichmüller space and the notion of $\lambda$-length introduced by Penner [Pe87]. In order to establish a connection between the two constructions, we derive a series of identities in hyperbolic geometry which we hope will be of interest in themselves.

To motivate our approach let us first recall some of the steps in the construction of the skein algebra and its relationship with the character variety. Let $q$ be the formal power series $e^{\frac{h}{4}} \in \mathbb{C}[[h]]$. The skein algebra $\mathcal{S}_{h}(\Sigma)$, introduced by Przytycki $[$ Pr91] and Turaev [Tu88], is the $\mathbb{C}[[h]]$-algebra generated by isotopy classes of framed links in $\Sigma \times[0,1]$ subject to the Kauffman bracket skein relation

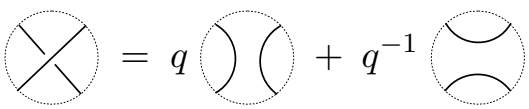

as well as the framing relation $\bigcirc=-q^{2}-q^{-2}$. In [Tu91], Turaev studied the relationship between the skein algebra and the Lie algebra of curves on $\Sigma$ introduced by Goldman [Go86]. In turn, in the work of Goldman, the Lie bracket on curves is related to the Weil-Petersson Poisson structure on the $S L_{2}(\mathbb{C})$-character variety $\mathcal{X}(\Sigma)$ of $\Sigma$, that is, the space of conjugacy classes of representations $\rho: \pi_{1}(\Sigma) \rightarrow S L_{2}(\mathbb{C})$. A direct relationship between the skein algebra and the character variety was described by Bullock [Bu97] who constructed a surjective homomorphism from the commutative algebra $\mathcal{S}_{0}(\Sigma)$ to the coordinate ring $\mathbb{C}[\mathcal{X}(\Sigma)]$ of $\mathcal{X}(\Sigma)$. This map turns out to be an isomorphism by the work of Przytycki and Sikora [PS00] (see more recently [CM09] for a direct proof). Up to a sign, it assigns to each free homotopy class of curves $\gamma$ in $\Sigma$ its trace function $t r_{\gamma}$ in $\mathbb{C}[\mathcal{X}(\Sigma)]$, given by taking the trace of representations evaluated at $\gamma$. One of the key ingredients is then given by the trace identities which relate the product of traces of two intersecting curves with the traces of their resolutions at one point. These identities, in turn, come from the classical formula $\operatorname{tr} A \cdot \operatorname{tr} B=\operatorname{tr} A B+\operatorname{tr} A B^{-1}$ relating traces in $S L_{2}(\mathbb{C})$. Using this isomorphism, Bullock, Frohman and KaniaBartoszyńska [BFK99] showed that the skein algebra is in fact a quantization of the character variety for the Goldman-Weil-Petersson bracket, in the sense of deformation of Poisson algebras. This means that $\mathcal{S}_{0}(\Sigma)$, endowed with the Poisson bracket inherited from the commutator on $\mathcal{S}_{h}(\Sigma)$, is isomorphic as a Poisson algebra to $\mathbb{C}[\mathcal{X}(\Sigma)]$.

Our goal is to extend this construction by including arcs in the definition of the skein algebra for a surface with punctures. We define a generalized link to be an embedding of a collection of circles and closed intervals in $\Sigma \times[0,1]$, so that the ends of the intervals sit above the punctures (see Section 2 for a 
precise definition).

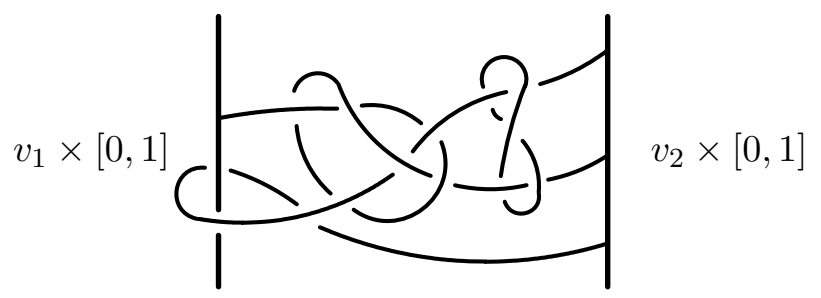

A component given by an interval will be called an arc. Instead of introducing framings, we follow the original approach of Kauffman [Kau90] and identify such links under a suitable notion of regular isotopy. The skein algebra of arcs and links $\mathcal{A S}_{h}(\Sigma)$ of $\Sigma$ will then be generated by regular isotopy classes of generalized links subject to the following set of relations. The usual skein relation still applies for crossings occurring above $\Sigma$, where we allow some of the strands to be arcs. When two arcs meet at a puncture we introduce the so-called puncture-skein relation

$$
\circlearrowleft=\frac{1}{v}\left(q^{\frac{1}{2}} \curvearrowright+q^{-\frac{1}{2}} \bigodot\right)
$$

Here $v$ is a central element associated to the puncture which turns out to be essential when trying to interpret this relation geometrically. In addition, the framing relation still applies and we also impose the puncture-framing relation $\odot=q+q^{-1}$.

In the classical case, we consider the algebra $\mathcal{C}(\Sigma)$ generated by arcs and loops on $\Sigma$ itself subject to the corresponding non-quantum skein relations. It admits a Poisson bracket described in terms of resolutions of intersections inside the surface and at the punctures which generalizes Goldman's Lie bracket on loops. Using arguments similar to the ones in [BFK99], we show that this bracket comes from the commutator in $\mathcal{A S}_{h}(\Sigma)$, In other words

Theorem 1.1. $\mathcal{A S}_{h}(\Sigma)$ is a deformation quantization of $\mathcal{C}(\Sigma)$.

The next step of our construction is to relate the algebra $\mathcal{C}(\Sigma)$ to the $S L_{2}-$ geometry of the surface $\Sigma$, in the case when $\chi(\Sigma)<0$ and the set of punctures $V$ is non-empty. In this context, the relevant object is the decorated Teichmüller space $\mathcal{T}^{d}(\Sigma)$ introduced by Penner [Pe87]. It is defined as a bundle over the usual Teichmüller space $\mathcal{T}(\Sigma)$ with fiber $\mathbb{R}_{>0}^{V}$. Given a hyperbolic metric $m \in \mathcal{T}(\Sigma)$, the choice of a point in the fiber corresponds to fixing the length of a horocycle at each of the punctures of $\Sigma$. This assignment, in turn, permits the measure of the length $l(\alpha)$ of any arc $\alpha$ between horocycles. A more relevant quantity in our context is the $\lambda$-length of $\alpha$ given by $\lambda(\alpha)=e^{\frac{l(\alpha)}{2}}$. This quantity satisfies the well-known Ptolemy relation

$$
\lambda(e) \lambda\left(e^{\prime}\right)=\lambda(a) \lambda(c)+\lambda(b) \lambda(d)
$$


where $a, b, c, d$ are the consecutive edges of a quadrilateral and $e$ and $e^{\prime}$ are its diagonals. Graphically, the Ptolemy relation can be rewritten:

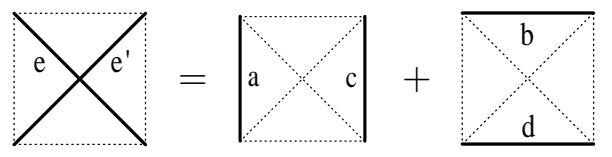

which we want to interpret as a non-quantum skein relation.

Using these notions, we obtain the following theorem.

Theorem 1.2. There is a well-defined homomorphism of Poisson algebras

$$
\Phi: \mathcal{C}(\Sigma) \rightarrow C^{\infty}\left(\mathcal{T}^{d}(\Sigma)\right)
$$

Up to signs, this map sends loops to their trace functions, arcs to their $\lambda$ lengths and punctures to horocycle lengths around them. The Poisson structure on $\mathcal{T}^{d}(\Sigma)$ is an extension of the usual Weil-Petersson Poisson bracket on $\mathcal{T}(\Sigma)$ and was described by Mondello [Mo09]. The proof of the theorem relies on a collection of length identities which generalize Penner's Ptolemy relation, the trace identities and Wolpert's cosine formula [Wo83] for the Poisson bracket of two trace functions. These identities are derived in turn from a set of "cosine laws" which can be found in Appendix A of [GL09] by Guo and Luo.

Combining Theorems 1.1 and 1.2 , it is tempting to interpret $\mathcal{A S}_{h}(\Sigma)$ as a deformation quantization of the decorated Teichmüller space. Following [Bu97] and [PS00], we first conjecture that the homomorphism $\Phi$ in the theorem above is injective. Another important step would then be to understand what is the correct "algebra of functions" on $\mathcal{T}^{d}(\Sigma)$. In their work, Bullock, Frohman and Kania-Bartoszyńska make use of the fact that the character variety is an algebraic variety, and as such they are lead to use its coordinate ring $\mathbb{C}[\mathcal{X}(\Sigma)]$. This choice is made natural by the fact that $\mathbb{C}[\mathcal{X}(\Sigma)]$ is generated by trace functions. In our context, the image of the homomorphism $\Phi$ is essentially the subalgebra generated by trace functions and $\lambda$-lengths. A natural question to ask is then if $\mathcal{T}^{d}(\Sigma)$ has a natural structure of an algebraic variety for which its coordinate ring coincides with this subalgebra. An important observation which makes this approach sensible is the fact that the $\lambda$-lengths associated to the edges of an ideal triangulation form a coordinate system on $\mathcal{T}^{d}(\Sigma)$ in which every trace function can be written as a Laurent polynomial (see Proposition 3.19 and Theorem 3.22). The way this fact translates at the level of the skein algebra $\mathcal{A S}_{h}(\Sigma)$, however, remains an intriguing problem.

The fact that the trace identity and the Ptolemy relation can be combined into generalized skein relations involving both arcs and loops has been used recently in works of Dupont and Palesi [DP11] and Musiker and Williams [MW11], in the context of cluster algebras associated to triangulated surfaces, and was observed previously by Fock and Goncharov [FG06]. It would be interesting to see if our work applies to the context of quantum cluster algebras as defined by Berenstein and Zelevinsky [BZ05]. Closely related to these considerations is the construction of quantum trace functions in the context of the 
quantization of Teichmüller space [Ka98, CF99]. This problem was solved recently by Bonahon and Wong in [BW10, BW11] using the skein relation in a crucial way. In turn, their construction is based on the use of shear coordinates [Bo96] which are closely related to $\lambda$-lengths. As such, we hope that our work could shed new light on the relationship between the skein algebra and the quantum Teichmüller space.

Acknowledgments. We are grateful to M. Freedman, C. Frohman, J. Przytycki and Z-H.Wang for showing interest in this work and for their helpful comments. We would also like to thank F. Bonahon and F. Luo for their continuous support and for the many conversations which have lead to this work. Finally, we would like to thank the referees for the very helpful suggestions on improving this article.

\section{Algebraic and topological aspects}

2.1. The skein algebra of arcs and links. We consider a surface $\Sigma$ obtained from a closed oriented surface $\bar{\Sigma}$ by removing a possibly empty finite subset $V$. Elements of $V$ will be called the punctures of $\Sigma$.

Definition 2.1. A continuous map $\alpha=\coprod_{i} \alpha_{i} \sqcup \coprod_{j} l_{j}$ from a finite collection of intervals $\coprod_{i}[0,1]$ and circles $\coprod_{j} S^{1}$ into $\bar{\Sigma} \times[0,1]$ is called a generalized link in $\Sigma \times[0,1]$ if

(1) $\alpha$ is an injection into $\bar{\Sigma} \times(0,1)$;

(2) each $l_{j}$ is an embedding into $\Sigma \times(0,1)$;

(3) the restriction of each $\alpha_{i}$ to $(0,1)$ is an embedding into $\Sigma \times(0,1)$;

(4) the restriction of each $\alpha_{i}$ to $\{0,1\}$ is an injection into $V \times(0,1)$.

We identify such embeddings under a change of orientation of any of their components.

Definition 2.2. Given two generalized links $\alpha$ and $\beta$ in $\Sigma \times[0,1]$ we define the stacking of $\alpha$ over $\beta$ to be the union in $\Sigma \times[0,1]$ of the rescaling of $\alpha$ to $\Sigma \times\left[\frac{1}{2}, 1\right]$ and the rescaling of $\beta$ to $\Sigma \times\left[0, \frac{1}{2}\right]$.

The open interval in condition (1) implies that the stacking of two generalized links is still injective, hence is a generalized link in $\Sigma \times[0,1]$. Each component $\alpha_{i}$ in a generalized link $\alpha$ will be called an arc, with the understanding that such a component can be "knotted", and condition (4) implies that each arc component of a link ends at a different height above the punctures of $\Sigma$.

Some conventions are needed when considering a diagram of a generalized link projected onto $\Sigma$. We use the usual convention to encode which strand of a generalized link passes over another in $\Sigma \times[0,1]$ and we assume that the diagram only possesses ordinary double points in $\Sigma$. However, we cannot impose this condition on the diagrams for intersections occurring above a puncture, since more than two arcs can meet at a puncture and such intersections cannot 
be resolved via an ambient isotopy of $\Sigma \times[0,1]$. If two strands of arcs meet above a puncture, we consider the following diagram:

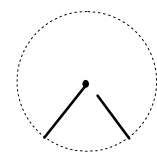

Here the left strand ends above the right one at the puncture, and no other strand ends in between. We call such a configuration a consecutive crossing. However, we do not necessarily sketch the strands ending above or below. In some cases, we need to study relations involving more than two strands ending at a puncture, then a picture such as this one,

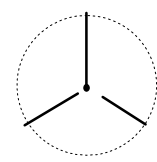

will be supplemented with an explanation of the respective positions of the strands lying under the top one.

Following the approach of Kauffman [Kau90], we say that two links are regularly isotopic if the diagram of one can be obtained from that of the other via consecutive applications of Reidemeister Moves II and III

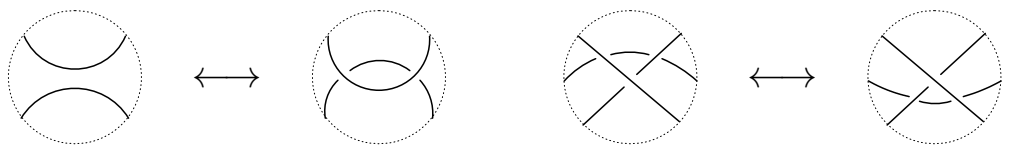

as well as the following new moves (Figure 1), occuring when two strands of arcs cross consecutively at a puncture, which we will call Reidemeister Moves $I I^{\prime}$. As is standard in the context of skein theory, we do not identify diagrams

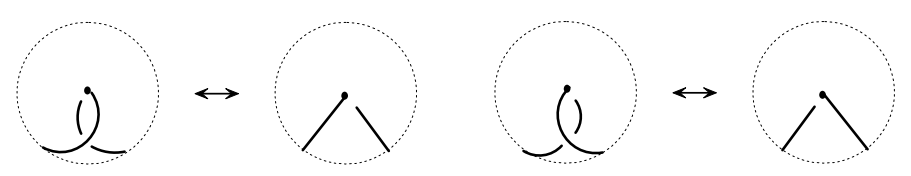

Figure 1. Reidemeister Moves II'.

under Reidemeister Moves I. We will show in Section 2.2 that Moves II, II' and III correspond to the moves needed when performing regular homotopies on the surface.

Let $\mathbb{C}[[h]]$ denote the ring of power series in $h$. We endow it with the $h-$ adic topology and, following the approach of Bullock, Frohman and KaniaBartoszyńska [BFK99] we will work over the category of topological $\mathbb{C}[[h]]$ modules. We refer to their paper as well as to the standard reference [Kas95] for details.

We let $\mathcal{L}$ be the set of regular isotopy classes of generalized links in $\Sigma \times$ $[0,1]$ together with the empty link and let $V^{ \pm 1}$ be the set of punctures $v$ of 
$\Sigma$ and their formal inverses $v^{-1}$. We consider the $\mathbb{C}$-vector space $\mathbb{C}\left[\mathcal{L}, V^{ \pm 1}\right]$ with basis $\mathcal{L} \cup V^{ \pm 1}$ and define a product on this space via

(1) the product $\alpha \cdot \beta$ of $\alpha$ and $\beta$ in $\mathcal{L}$ is obtained by stacking $\alpha$ over $\beta$;

(2) the elements of $V^{ \pm 1}$ are central and $v \cdot v^{-1}=1$ for each $v \in V$.

The empty link is the identity for this operation.

We can then form the set $\mathbb{C}\left[\mathcal{L}, V^{ \pm 1}\right][[h]]$ of formal power series with coefficients in $\mathbb{C}\left[\mathcal{L}, V^{ \pm 1}\right]$ which inherits a natural structure of $\mathbb{C}[[h]]$-module. The multiplication on $\mathbb{C}\left[\mathcal{L}, V^{ \pm 1}\right]$ extends naturally to $\mathbb{C}\left[\mathcal{L}, V^{ \pm 1}\right][[h]]$ and turns it into a topological algebra.

We are now ready to introduce the main object of this article.

Definition 2.3. Let $q$ be the formal power series $e^{\frac{h}{4}} \in \mathbb{C}[[h]]$. The skein algebra of arcs and links $\mathcal{A S}_{h}(\Sigma)$ is the quotient of $\mathbb{C}\left[\mathcal{L}, V^{ \pm 1}\right][[h]]$ by the closure in the $h$-adic topology of the sub-module generated by the following relations:

(1) Kauffman Bracket Skein Relation: for a crossing in the surface, we have

$$
\circlearrowright=q \bigcirc\left(q^{-1} \circlearrowright\right.
$$

(2) Puncture-Skein Relation: for a consecutive crossing at a puncture $v$, we have

$$
\zeta=\frac{1}{v}\left(q^{\frac{1}{2}} \curvearrowright+q^{-\frac{1}{2}} \bigodot\right)
$$

(3) Framing Relation: for the isotopy class of a trivial loop, we have

$$
\bigcirc=-q^{2}-q^{-2}
$$

(4) Puncture-Framing Relation: for the isotopy class of a circle around a puncture, we have

$$
\text { ○ }=q+q^{-1} \text {. }
$$

The multiplication $\cdot$ is induced by the stacking operation on $\mathbb{C}\left[\mathcal{L}, V^{ \pm 1}\right][[h]]$.

Some comments are in order to justify this definition. First, note that if $V$ is empty, then $\mathcal{A S}_{h}(\Sigma)$ coincides with the algebra defined in [BFK99], which is a topological version of the usual Kauffman bracket skein algebra $\mathcal{S}_{q}(\Sigma)$ over a formal parameter $q$ as defined in [Pr91] and [Tu88]. Second, The choice of the coefficients $q^{ \pm \frac{1}{2}}$ in the puncture-skein relation turns out to be essential in proving that this algebra is well-defined and that the product is associative. It will also have a geometrical justification which will be explained in Section 3. Finally, the central elements $v$ associated to the punctures are not essential from the algebraic point of view but will play an important rôle in the geometric interpretation given in Section 3, where they will be related to the choice of a horocycle at each puncture. 
We recall that a $\mathbb{C}[[h]]-$ module $M$ is called topologically free if there exists a $\mathbb{C}$-vector space $\mathcal{V}$ so that $M$ is isomorphic to $\mathcal{V}[[h]]$ (see for example [Kas95]). We have the following

Theorem 2.4. The skein algebra $\left(\mathcal{A S}_{h}(\Sigma), \cdot\right)$ is a well-defined topologically free associative $\mathbb{C}[[h]]$-algebra.

Proof. One can easily see that the stacking operation is compatible with regular isotopies. Therefore, to verify the well-definedness of the multiplication, it suffices to show that it is invariant under Reidemeister Moves II, II and III. The invariance under Reidemeister Moves II and III follows from the same arguments as in [Pr91]. For Reidemeister Move II', we first calculate that

$$
\begin{aligned}
\circlearrowleft= & \frac{1}{v}\left(q^{\frac{1}{2}} \dot{\bigcirc}+q^{-\frac{1}{2}} \odot\right) \\
& =\frac{1}{v}\left(q^{\frac{1}{2}}\left(-q^{2}-q^{-2}\right)+q^{-\frac{1}{2}}\left(q+q^{-1}\right)\right)=\frac{1}{v}\left(q^{\frac{1}{2}}-q^{\frac{5}{2}}\right),
\end{aligned}
$$

where $v$ is the puncture and the second equality follows from the framing and the puncture-framing relations. With this, we obtain

$$
\begin{aligned}
& \text { (i) }=q\left(q^{-1} 0\right. \\
& =\frac{1}{v} q\left(q^{\frac{1}{2}} \stackrel{\circ}{\bigcirc}+q^{-\frac{1}{2}} \curvearrowright\right)+\frac{1}{v} q^{-1}\left(q^{\frac{1}{2}}-q^{\frac{5}{2}}\right) \bigcirc \\
& =\frac{1}{v}\left(q^{\frac{1}{2}} \curvearrowright+q^{-\frac{1}{2}} \bigcirc\right)=\circlearrowright
\end{aligned}
$$

where the first equality follows from the Kauffman bracket skein relation and the second equality from the puncture-skein relation and the previous calculation. The well-definedness under the other Reidemeister Move II' is verified similarly.

To show that $\alpha \cdot \odot=\bigodot \cdot \alpha=\left(q+q^{-1}\right) \alpha$, the only case we need to consider is when $\odot$ is a circle around a puncture $v$ and $\alpha$ is an arc having $v$ as one of its endpoints. For $\alpha \cdot \odot$, we have

$$
0=q \Omega\left(q+q^{-1}\right)=(Q)
$$

and similarly for $\odot \cdot \alpha=\left(q+q^{-1}\right) \alpha$.

When three links cross inside the surface, the associativity follows from the same arguments as in [Pr91], and similarly if some intersections happen at a puncture as long as there are no triple points. If three arcs $\alpha, \beta$ and $\gamma$ meet at 
a puncture $v$, say in counterclockwise order, we have for $(\alpha \cdot \beta) \cdot \gamma$ that

$$
\begin{aligned}
& \beta \zeta\rangle_{\gamma}^{\alpha}=\frac{1}{v}\left(q^{\frac{1}{2}} \circlearrowright+q^{-\frac{1}{2}} \circlearrowright\right) \\
& =\frac{1}{v}\left(q^{\frac{3}{2}} \bigcirc+q^{-\frac{1}{2}} \square+q^{-\frac{1}{2}} \circlearrowright\right) \text {; }
\end{aligned}
$$

and for $\alpha \cdot(\beta \cdot \gamma)$ that

$$
\begin{aligned}
& \beta \zeta\rangle_{\gamma}^{\alpha}=\frac{1}{v}\left(q^{\frac{1}{2}}(T)+q^{-\frac{1}{2}} \longrightarrow\right) \\
& =\frac{1}{v}\left(q^{\frac{3}{2}}\left(\backslash+q^{-\frac{1}{2}} \circlearrowright+q^{-\frac{1}{2}} \square\right)\right. \text {. }
\end{aligned}
$$

The case when $\alpha, \beta$ and $\gamma$ are ordered clockwise can be checked similarly.

The proof that $\mathcal{A S}_{h}(\Sigma)$ is topologically free follows from the same arguments as in [BFK99]. In our case, a diagram of a generalized link in $\Sigma \times[0,1]$ is a graph in $\bar{\Sigma}$ which is four-valent in $\Sigma$ and many-valent at $V$ with over- and under-crossings. Two diagrams represent the regularly isotopic generalized links if and only if they differ by a sequence of isotopies of $\Sigma$ and Reidemeister Moves II, II ${ }^{\prime}$ and III. We consider the vector space $\mathcal{W}$ over $\mathbb{C}$ whose basis consists of all diagrams which have no crossing in $\bar{\Sigma}$, no trivial loops and no loops bounding a puncture, and let $\mathcal{V}=\mathcal{W} \otimes \mathbb{C}\left[V^{ \pm 1}\right]$. To any element in $\mathbb{C}\left[\mathcal{L}, V^{ \pm 1}\right]$ one can associate an element of $\mathcal{V}[[h]]$ by considering one of its associated diagrams and first resolving intersections at the punctures using the puncture-skein relation (2), then resolving intersections in $\Sigma$ using the skein relation (1) and finally sending each trivial loops to $-q^{2}-q^{-2}$ and loops around punctures to $q+q^{-1}$. This process converges when extended to power series in $\mathbb{C}\left[\mathcal{L}, V^{ \pm 1}\right][[h]]$ and can be seen to descend to a well-defined homomorphism of topological algebras $\Psi: \mathcal{A S}_{h}(\Sigma) \rightarrow \mathcal{V}[[h]]$ whose inverse is given by considering the inclusion of $\mathcal{V}[[h]]$ in $\mathbb{C}\left[\mathcal{L}, V^{ \pm 1}\right][[h]]$ and taking the quotient.

q.e.d.

Remark 2.5. In the rest of this paper, we call an element $\mathcal{S}$ of $\mathcal{V}=\mathcal{W} \otimes$ $\mathbb{C}\left[V^{ \pm 1}\right]$ a state. Recall that $\mathcal{W}$ consists of all the diagrams on $\Sigma$ with no crossings and no loops bounding a disk or a puncture.

2.2. Generalized curves and regular homotopy. The classical counterpart of the skein algebra can be defined in terms of curves on the surface $\Sigma$ itself. As such we start by introducing the proper class of curves we will need to consider.

Definition 2.6. A generalized curve on $\Sigma$ is an immersion $\alpha: D \rightarrow \bar{\Sigma}$ of a finite union $D$ of circles and closed intervals into $\bar{\Sigma}$ so that

(1) the circles and the interior of the intervals are mapped into $\Sigma$;

(2) the end points of the intervals are mapped to the set of punctures $V$. 
We identify such immersions under a change of orientation of any of their components.

The empty map is also considered as a generalized curve and called the empty curve.

Definition 2.7. Two generalized curves $\alpha$ and $\beta: D \rightarrow \bar{\Sigma}$ are called regularly homotopic if there exists a homotopy $F: D \times[0,1] \rightarrow \bar{\Sigma}$ from $\alpha$ to $\beta$ relative to the end points of the intervals so that $\left.F\right|_{D \times\{t\}}$ is an immersion for all $t \in[0,1]$.

We refer to [Wh37] for more details of regular homotopies. Besides the standard Reidemeister Moves II and III,

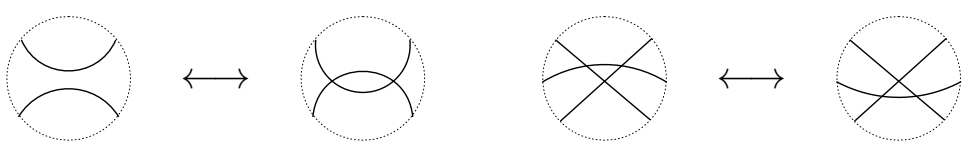

we also introduce the Reidemeister Move II' for generalized curves:

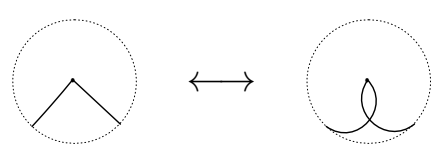

Lemma 2.8. Two generalized curves on $\Sigma$ are regularly homotopic if and only if one can be obtained from the other by a sequence of ambient isotopies of $\Sigma$ and Reidemeister Moves II, II' and III.

Proof. If two generalized curves differ by a Reidemeister Move II, II' or III, then we can perform the following regular homotopies or their inverses respectively:

(II) For a Reidemeister Move II, the homotopy creates a tangential double point where the two strands of the curve intersect tangentially;

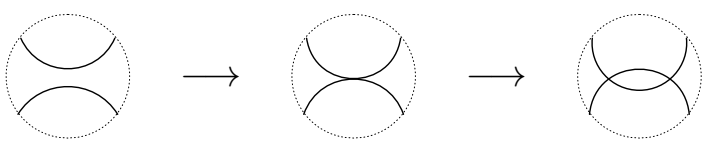

(II') For a Reidemeister Move II', the homotopy creates a tangential double point at a puncture where the two ends intersect tangentially;

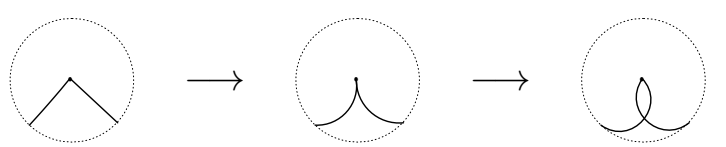

(III) For a Reidemeister Move III, the homotopy creates a transverse triple point where the three strands intersect.

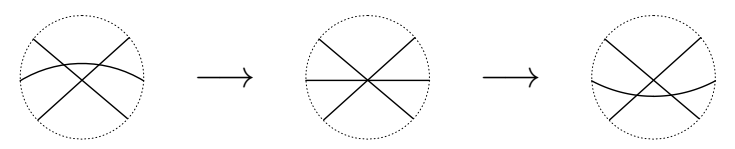


For the converse, we follow the approach of Goldman in [Go86, 5.6. Lemma]. Let $\operatorname{Imm}(D, \bar{\Sigma})$ be the space of generalized curves $\alpha: D \rightarrow \bar{\Sigma}$ endowed with the Fréchet topology, and we call a generalized curve $\alpha: D \rightarrow \bar{\Sigma}$ generic if its only self-intersections in $\Sigma$ are transverse double points and its self-intersections at the punctures $v \in V$ are all transverse. By standard transversality arguments (see for example [Hi76]), generic immersions form an open dense subset $\operatorname{Imm}_{0}(D, \bar{\Sigma})$ of $\operatorname{Imm}(D, \bar{\Sigma})$. Denote by $\operatorname{Imm}_{1}(D, \bar{\Sigma})$ the subspace of $\operatorname{Imm}(D, \bar{\Sigma})$ consisting of immersions $\alpha: D \rightarrow \bar{\Sigma}$ with finitely many transverse double points and exactly one of the following singularities:
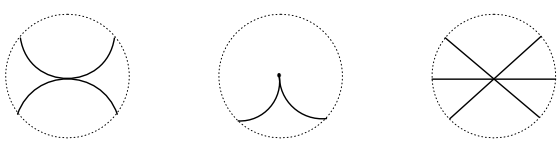

Then, by transversality, $\operatorname{Imm}_{1}(D, \bar{\Sigma})$ has codimension 1 in $\operatorname{Imm}(D, \bar{\Sigma})$.

Suppose now that $\alpha$ and $\beta$ are two regularly homotopic generalized curves in $\Sigma$. By transversality, they are homotopic via a generic path in $\operatorname{Im} m(D, \bar{\Sigma})$, that is, a path which avoids entirely the codimension $\geqslant 2$ subset $\operatorname{Imm}(D, \bar{\Sigma}) \backslash$ $\left(\operatorname{Imm}_{0}(D, \bar{\Sigma}) \cup \operatorname{Imm}_{1}(D, \bar{\Sigma})\right)$ and meets $\operatorname{Imm}_{1}(D, \bar{\Sigma})$ transversely. Thus, there is a homotopy $F_{t}$ from $\alpha$ to $\beta$ such that $F_{t} \in \operatorname{Imm}_{0}(D, \bar{\Sigma})$ for all $t \in[0,1]$ except at finitely many $t_{i} \in\left\{t_{1}, \ldots, t_{k}\right\}$ where $F_{t_{i}} \in \operatorname{Imm}_{1}(D, \bar{\Sigma})$ and the homotopy $F_{t}$ in a neighborhood of $t_{i}$ is of one of the types described in (II), (II') or (III) above.

q.e.d.

2.3. The Poisson algebra of generalized curves. In this section, we introduce the classical counterpart $\mathcal{C}(\Sigma)$ of the skein algebra $\mathcal{A S}_{h}(\Sigma)$ and show that one is a deformation quantization of the other.

Definition 2.9. The algebra of curves $\mathcal{C}(\Sigma)$ on $\Sigma$ is the quotient of the $\mathbb{C}$ algebra generated by the regular homotopy classes of generalized curves on $\Sigma$, the punctures of $\Sigma$ and their formal inverses, modulo the subspace generated by the following relations:

$\left(1^{\prime}\right)$ Skein Relation: $X=\Omega 0+\infty$ for an intersection in $\Sigma$

$\left(2^{\prime}\right)$ Puncture-Skein Relation: $\triangle=v^{-1}(\curvearrowright+\infty)$ for an intersection at $v$;

$\left(3^{\prime}\right)$ Framing Relation: $\bigcirc=-2$;

(4') Puncture-Framing Relation: $\odot=2$.

The product $\alpha \cdot \beta$ of two generalized curves $\alpha$ and $\beta$ is obtained by taking their union with unit the empty curve.

The fact that $\mathcal{C}(\Sigma)$ is a well-defined commutative algebra follows from the same arguments as for $\mathcal{A S}_{h}(\Sigma)$. These two algebras are related naturally as 
follows: let $p: \mathcal{A S}_{h}(\Sigma) \rightarrow \mathcal{C}(\Sigma)$ be the map which to a generalized link in $\Sigma \times[0,1]$ associates its projection on $\Sigma$. We also let $p(h)=0$ and let $p\left(v^{ \pm 1}\right)=$ $v^{ \pm 1}$ for each puncture $v$. Since $p$ maps regular isotopies to regular homotopies and relations $(1)-(4)$ to the corresponding relations $\left(1^{\prime}\right)-\left(4^{\prime}\right)$, and maps the stacking of generalized framed links in $\Sigma \times[0,1]$ to the union of generalized curves on $\Sigma$, it is a well-defined surjective $\mathbb{C}$-algebra homomorphism.

Proposition 2.10. The map $\bar{p}: \mathcal{A S}_{h}(\Sigma) / h \mathcal{A S}_{h}(\Sigma) \rightarrow \mathcal{C}(\Sigma)$ induced by $p$ is an isomorphism of $\mathbb{C}$-algebras.

Proof. Since $\mathcal{A S}_{h}(\Sigma) \cong \mathcal{V}[[h]]$ is topologically free, each element $a \in$ $\mathcal{A S}_{h}(\Sigma)$ can be uniquely written as a power series $\sum a_{k} h^{k}$ with coefficients $a_{i} \in \mathcal{V}$. By the definition of $p$, we have $p(a)=p\left(a_{0}\right)$. Remember that the elements of $\mathcal{V}$ are diagrams without crossings either in $\Sigma$ or at $V$, hence $p$ is injective on $\mathcal{V}$. Since $a_{0} \in \mathcal{V}$, we have $p\left(a_{0}\right)=0$ if and only if $a_{0}=0$. As a consequence, $\operatorname{ker} p=h \mathcal{A S}_{h}(\Sigma)$ and $p$ induces a $\mathbb{C}$-algebra isomorphism $\bar{p}: \mathcal{A S}_{h}(\Sigma) / h \mathcal{A S}_{h}(\Sigma) \rightarrow \mathcal{C}(\Sigma)$.

q.e.d.

In [Go86], Goldman defines a Lie bracket on the free algebra generated by free homotopy classes of curves on $\Sigma$. It can be described in terms of resolutions of intersections and is of a purely topological nature. Generalizing this construction, we consider the Goldman bracket on $\mathcal{C}(\Sigma)$ to be the bilinear $\operatorname{map}\{\}:, \mathcal{C}(\Sigma) \times \mathcal{C}(\Sigma) \rightarrow \mathcal{C}(\Sigma)$ defined as follows:

(1) for two punctures $v$ and $w$, we let $\{v, w\}=0$;

(2) for a puncture $v$ and a generalized curve $\alpha$, we let $\{v, \alpha\}=0$;

(3) for two generalized curves $\alpha$ and $\beta$, we let

$$
\{\alpha, \beta\}=\frac{1}{2} \sum_{p \in \alpha \cap \beta \cap \Sigma}\left(\alpha_{p} \beta^{+}-\alpha_{p} \beta^{-}\right)+\frac{1}{4} \sum_{v \in \alpha \cap \beta \cap V} \frac{1}{v}\left(\alpha_{v} \beta^{+}-\alpha_{v} \beta^{-}\right) .
$$

In the first sum, the positive resolution $\alpha_{p} \beta^{+}$of $\alpha$ and $\beta$ at $p$ is obtained by going along $\alpha$ toward $p$ then turning left at $p$ before going along $\beta$, and the negative resolution $\alpha_{p} \beta^{-}$is obtained similarly by turning right.
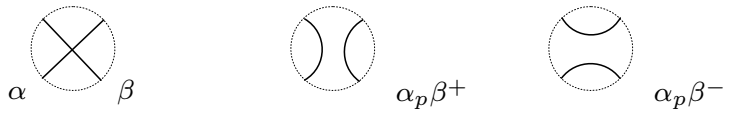

In the second sum, we introduce positive and negative resolutions of intersections at a puncture in a similar manner. However, we have to consider several cases depending on the number and the relative positions of the ends of $\alpha$ and $\beta$ meeting at $v$. Given an end of $\alpha$ and an end of $\beta$ at $v$, a positive resolution consists in going along the corresponding strand of $\alpha$ toward $v$ then turning left around $v$ before going along the strand of $\beta$. In this process the other ends of $\alpha$ and $\beta$, if any, are left untouched. The positive resolution $\alpha_{v} \beta^{+}$ is then defined to be the sum of all the positive resolutions between the ends of $\alpha$ and the ends of $\beta$. The negative resolution $\alpha_{v} \beta^{-}$is defined accordingly by turning right around $v$. The possible configurations and resolutions are given in the table below. 


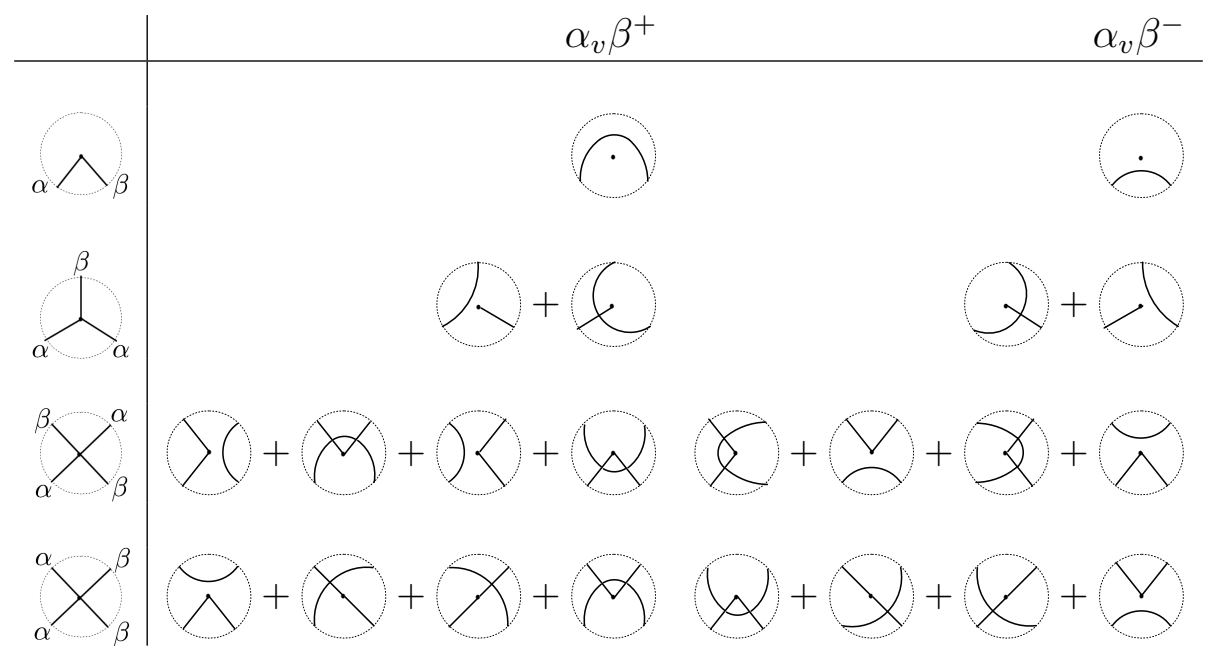

Similarly to the skein algebra, most of the facts about this bracket rely on computations done locally around intersections. As such, diagrams of these types

$$
\left\{(i)_{\beta}\right\} \quad\{\overbrace{\alpha}^{\beta}\}
$$

will be used to denote the sum of the terms in $\{\alpha, \beta\}$ coming from their intersections in the dotted circle. Note in particular that the order in the bracket will be encoded using over and under crossings, even though the curves do intersect in $\bar{\Sigma}$.

We recall that a Poisson algebra is an associative algebra together with an anti-symmetric bilinear form which satisfies the Jacobi identity as well as the Leibniz rule.

Theorem 2.11. The algebra $(\mathcal{C}(\Sigma), \cdot,\{\}$,$) is a well-defined Poisson alge-$ bra.

To prove Theorem 2.11, we need the following lemma.

Lemma 2.12. The following identities hold in $\mathcal{C}(\Sigma)$ :

(1) $Q=-C$;

(2) $0=0$;

(3)

$$
2=\curvearrowright+2 \bigodot .
$$

Proof. This is a simple computation using the relations in Definition 2.9. As an illustration, for relation (2) we have 


$$
\left.\bigcirc=\frac{1}{v}(\odot)+\dot{\bigcirc}\right)=\frac{1}{v}(2-2)=0 \text {. }
$$

q.e.d.

Proof of Theorem 2.11. By Lemma 2.8, in order to verify the well-definedness, it suffices to show that $\{$,$\} is invariant under Reidemeister Moves II, II' and$ III and relations $\left(1^{\prime}\right)-\left(4^{\prime}\right)$. The invariance under Reidemeister Moves II and III and relations $\left(1^{\prime}\right),\left(3^{\prime}\right)$ and $\left(4^{\prime}\right)$ follows from the same arguments as in [Go86]. For the invariance under Reidemeister Move II', We have, with the pictorial conventions set earlier,

$$
\begin{aligned}
& \left.\{\alpha)_{\beta}\right\}=\frac{1}{2}(\triangle-O)+\frac{1}{4 v}(\dot{l}-\mathcal{Q}) \\
& \left.\left.=\frac{1}{2 v}(\bigodot)+\curvearrowright\right)+\frac{1}{4 v}(-3 \bigcirc)-\curvearrowright\right) \\
& =\frac{1}{4 v}(\curvearrowright-\bigcirc)=\{\circlearrowleft\} \text {, }
\end{aligned}
$$

where the second equality follows from Lemma 2.12. For the invariance under the puncture-skein relation $\left(3^{\prime}\right)$, we have to verify the following three cases:

(i) $\left\{\bigcap_{\alpha}^{\beta}\right\}=v^{-1}(\{\curvearrowright\}+\{\square\})$,

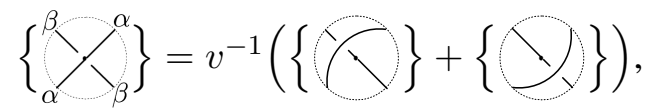

(iii) $\left\{>_{\alpha}^{\alpha}\right\}=v^{-1}(\{\Omega\}+\{\Omega\langle\})$.

For (i), if we apply to the left hand side the definition of the bracket then resolve the intersections in the surface using the skein relation, we obtain

$$
\begin{aligned}
& \left\{\bigcap_{\alpha}^{\beta}\right\}=\frac{1}{4 v}(\circlearrowright)+(X) \\
& =\frac{1}{4 v}(\Omega+\square+O-\square-Q(O) \\
& =\frac{1}{2 v}(\circlearrowright->)
\end{aligned}
$$

while by definition the second term on the right hand side vanishes and so

$$
\frac{1}{v}\left(\{\bigcirc)+\{\Longrightarrow)=\frac{1}{2 v}(\supset-C)\right. \text {. }
$$

Note that since the left hand sides of (ii) and (iii) differ by a Reidemeister Move $\mathrm{II}^{\prime}$, it suffices to verify either of them, which follows from a computation similar to that for (i) and is left to the reader. The anti-symmetry of $\{$, 
follows from the fact that $\alpha_{x} \beta^{ \pm}=\beta_{x} \alpha^{\mp}$ for each $x \in \alpha \cap \beta$ either in the surface or at the punctures.

The verification of the Jacobi identity is in the spirit of Goldman [Go86] separating the following two cases:

(1) $\alpha \cap \beta \cap \gamma \cap V=\emptyset$, and

(2) $\alpha \cap \beta \cap \gamma \cap V \neq \emptyset$.

In case (1), we let $\alpha, \beta$ and $\gamma$ be three generalized curves on $\Sigma$. We let $c(x, y)=\frac{1}{4}$ if $x, y \in \Sigma$ and $c(x, y)=\frac{1}{16} x^{-1} y^{-1}$ if $x, y \in V$; and if only one of $x$ and $y$, say $x$, is a puncture of $\Sigma$, we let $c(x, y)=\frac{1}{8} x^{-1}$. Then we have

$$
\begin{aligned}
& \{\{\alpha, \beta\}, \gamma\} \\
= & \sum_{\substack{x \in \alpha \cap \beta \\
y \in \beta \cap \gamma}} c(x, y)\left(\left(\alpha_{x} \beta^{+}\right)_{y} \gamma^{+}-\left(\alpha_{x} \beta^{+}\right)_{y} \gamma^{-}-\left(\alpha_{x} \beta^{-}\right)_{y} \gamma^{+}+\left(\alpha_{x} \beta^{-}\right)_{y} \gamma^{-}\right) \\
+ & \sum_{\substack{x \in \alpha \cap \beta \\
z \in \gamma \cap \alpha}} c(x, z)\left(\left(\alpha_{x} \beta^{+}\right)_{z} \gamma^{+}-\left(\alpha_{x} \beta^{+}\right)_{z} \gamma^{-}-\left(\alpha_{x} \beta^{-}\right)_{z} \gamma^{+}+\left(\alpha_{x} \beta^{-}\right)_{z} \gamma^{-}\right),
\end{aligned}
$$

and

$$
\begin{aligned}
& \{\{\beta, \gamma\}, \alpha\} \\
= & \sum_{\substack{y \in \beta \cap \gamma \\
z \in \gamma \cap \alpha}} c(y, z)\left(\left(\beta_{y} \gamma^{+}\right)_{z} \alpha^{+}-\left(\beta_{y} \gamma^{+}\right)_{z} \alpha^{-}-\left(\beta_{y} \gamma^{-}\right)_{z} \alpha^{+}+\left(\beta_{y} \gamma^{-}\right)_{z} \alpha^{-}\right) \\
+ & \sum_{\substack{y \in \beta \cap \gamma \\
x \in \alpha \cap \beta}} c(y, x)\left(\left(\beta_{y} \gamma^{+}\right)_{x} \alpha^{+}-\left(\beta_{y} \gamma^{+}\right)_{x} \alpha^{-}-\left(\beta_{y} \gamma^{-}\right)_{x} \alpha^{+}+\left(\beta_{y} \gamma^{-}\right)_{x} \alpha^{-}\right) .
\end{aligned}
$$

By definition, we have that $\left(\alpha_{x} \beta^{+}\right)_{y} \gamma^{+}=\left(\beta_{y} \gamma^{+}\right)_{x} \alpha^{-},\left(\alpha_{x} \beta^{+}\right)_{y} \gamma^{-}=$ $\left(\beta_{y} \gamma^{-}\right)_{x} \alpha^{-},\left(\alpha_{x} \beta^{-}\right)_{y} \gamma^{+}=\left(\beta_{y} \gamma^{+}\right)_{x} \alpha^{+}$and $\left(\alpha_{x} \beta^{-}\right)_{y} \gamma^{-}=\left(\beta_{y} \gamma^{-}\right)_{x} \alpha^{+}$for each $x \in \alpha \cap \beta$ and $y \in \beta \cap \gamma$, so the summands in the first row of the expansion of $\{\{\alpha, \beta\}, \gamma\}$ cancel out the summands in the second row of the expansion of $\{\{\beta, \gamma\}, \alpha\}$. Similarly, the summands in the second row of the expansion of $\{\{\alpha, \beta\}, \gamma\}$ and the first row of the expansion of $\{\{\beta, \gamma\}, \alpha\}$ cancel out the summands in the expansion of $\{\{\gamma, \alpha\}, \beta\}$. Hence $\{\{\alpha, \beta\}, \gamma\}+$ $\{\{\beta, \gamma\}, \alpha\}+\{\{\gamma, \alpha\}, \beta\}=0$. In case (2), we let $v \in \alpha \cap \beta \cap \gamma$. If $v$ is a self-intersection of one of $\alpha, \beta$ or $\gamma$, say $\alpha$, then by the well-definedness of $\{$,$\} we can resolve \alpha$ at $v$ to reduce to case (1). If $v$ is a self-intersection of none of $\alpha, \beta$ or $\gamma$, then we may without loss of generality assume that $\alpha, \beta$ and $\gamma$ are counterclockwise ordered at $v$. Then all the summands in $\{\{\alpha, \beta\}, \gamma\}+$ $\{\{\beta, \gamma\}, \alpha\}+\{\{\gamma, \alpha\}, \beta\}$ cancel out in pairs as in case (1) except three summands around $v$ which are from $\frac{1}{4} v^{-1}\left\{\alpha_{v} \beta^{+}, \gamma\right\}, \frac{1}{4} v^{-1}\left\{\beta_{v} \gamma^{+}, \alpha\right\}$ and 
$\frac{1}{4} v^{-1}\left\{\gamma_{v} \alpha^{+}, \beta\right\}$ respectively; and for the sum of them, we have

$$
\begin{aligned}
& \{C\}+\{C\}+\{C\} \\
& =\frac{1}{2}(\zeta-\square)+\frac{1}{2}(\supset)+\frac{1}{2}(\square-\circlearrowright)=0 \text {. }
\end{aligned}
$$

The Leibniz rule follows directly from the definition and the fact that $(\alpha \cdot \beta) \cap$ $\gamma=(\alpha \cup \beta) \cap \gamma=(\alpha \cap \gamma) \cup(\beta \cap \gamma)$.

q.e.d.

Following the approach of [BFK99] (see also [KS98]), we recall that a topologically free $\mathbb{C}[[h]]$-algebra $A_{h}$ is called a (formal) deformation quantization of a Poisson algebra $A$ if there is a $\mathbb{C}$-algebra isomorphism $\Theta: A_{h} / h A_{h} \rightarrow A$ such that

$$
\Theta\left(\frac{\bar{\alpha} \cdot \bar{\beta}-\bar{\beta} \cdot \bar{\alpha}}{h}\right)=\{\alpha, \beta\}
$$

for any $\bar{\alpha} \in \Theta^{-1}(\alpha)$ and $\bar{\beta} \in \Theta^{-1}(\beta)$. Using the isomorphism from Proposition 2.10, we obtain the following theorem.

Theorem 2.13. The $\mathbb{C}[[h]]$-algebra $\mathcal{A S}_{h}(\Sigma)$ is a deformation quantization of $\mathcal{C}(\Sigma)$ via the $\mathbb{C}$-algebra isomorphism $\bar{p}$.

Proof. We adapts arguments which can be found in [BFK99]. Given a diagram on the surface, we let $p^{ \pm}(\mathcal{S})$, respectively, be the number of positive and negative resolutions in the surface used to obtain the state $\mathcal{S}$, and let $v^{ \pm}(\mathcal{S})$, respectively, be the number of positive and negative resolutions at the punctures used to obtain $\mathcal{S}$. If none of $\alpha$ and $\beta$ has a self-intersection at the punctures, then, keeping track of the crossings, we have

$$
\{\alpha, \beta\}=\sum_{\mathcal{S}}\left(\frac{1}{2}\left(p^{+}(\mathcal{S})-p^{-}(\mathcal{S})\right)+\frac{1}{4}\left(v^{+}(\mathcal{S})-v^{-}(\mathcal{S})\right)\right) \mathcal{S},
$$

where the summation is taken over all states $\mathcal{S}$ obtained from resolving $\alpha \cup \beta$, and

$$
\begin{aligned}
\bar{\alpha} \cdot \bar{\beta}-\bar{\beta} \cdot \bar{\alpha}=\sum_{\mathcal{S}}( & q^{\left(p^{+}(\mathcal{S})-p^{-}(\mathcal{S})\right)+\frac{1}{2}\left(v^{+}(\mathcal{S})-v^{-}(\mathcal{S})\right)} \\
& \left.-q^{-\left(p^{+}(\mathcal{S})-p^{-}(\mathcal{S})\right)-\frac{1}{2}\left(v^{+}(\mathcal{S})-v^{-}(\mathcal{S})\right)}\right) \mathcal{S},
\end{aligned}
$$

in which the coefficient of $h$ is exactly $\{\alpha, \beta\}$. If one of $\alpha$ or $\beta$, say $\alpha$, has a self-intersection at a puncture $v \in V$, then we let $\alpha_{1}$ and $\alpha_{2}$ be the resolutions of $\alpha$ at $v$. Let $\sigma_{1}$ and $\sigma_{2}$ respectively be the set of states obtained by resolving $\alpha_{1} \cup \beta$ and $\alpha_{2} \cup \beta$, and let $n_{\mathcal{S}}=\frac{1}{2}\left(p^{+}(\mathcal{S})-p^{-}(\mathcal{S})\right)+\frac{1}{4}\left(v^{+}(\mathcal{S})-v^{-}(\mathcal{S})\right)$. By the previous calculation, we have

$$
\{\alpha, \beta\}=\frac{1}{v}\left(\left\{\alpha_{1}, \beta\right\}+\left\{\alpha_{2}, \beta\right\}\right)=\frac{1}{v} \sum_{\mathcal{S} \in \sigma_{1} \cup \sigma_{2}} n_{\mathcal{S}} \mathcal{S} .
$$


If $\bar{\alpha}_{1}$ and $\bar{\alpha}_{2}$, respectively, are the positive and negative resolutions of $\bar{\alpha}$ at $v$, then by the puncture-skein relation $\bar{\alpha}=v^{-1}\left(q^{\frac{1}{2}} \bar{\alpha}_{1}+q^{-\frac{1}{2}} \bar{\alpha}_{2}\right)$, and we have that

$$
\begin{aligned}
\bar{\alpha} \cdot \bar{\beta}-\bar{\beta} \cdot \bar{\alpha} & =\frac{1}{v}\left(q^{\frac{1}{2}}\left(\bar{\alpha}_{1} \cdot \bar{\beta}-\bar{\beta} \cdot \bar{\alpha}_{1}\right)+q^{-\frac{1}{2}}\left(\bar{\alpha}_{2} \cdot \bar{\beta}-\bar{\beta} \cdot \bar{\alpha}_{2}\right)\right) \\
& =\frac{1}{v}\left(q^{\frac{1}{2}} \sum_{\mathcal{S} \in \sigma_{1}}\left(q^{2 n_{\mathcal{S}}}-q^{-2 n_{\mathcal{S}}}\right) \mathcal{S}+q^{-\frac{1}{2}} \sum_{\mathcal{S} \in \sigma_{2}}\left(q^{2 n_{\mathcal{S}}}-q^{-2 n_{\mathcal{S}}}\right) \mathcal{S}\right)
\end{aligned}
$$

in which the coefficient of $h$ is $\{\alpha, \beta\}$.

q.e.d.

In particular, the proof of Theorem 2.13 explains the relationship between the coefficients $q^{ \pm \frac{1}{2}}$ in the puncture-skein relation used in the definition of $\mathcal{A S}_{h}(\Sigma)$ and the coefficient $\frac{1}{4}$ in front of the puncture terms in the Goldman bracket on $\mathcal{C}(\Sigma)$. Both of these choices were essential at some point in the well-definedness of $\mathcal{A S}_{h}(\Sigma)$ and $\{$,$\} and turn out to be related to the geo-$ metric aspects of the theory described in the next section.

\section{Relationship with hyperbolic geometry}

An essential aspect of the skein algebra is its relationship with the $S L_{2}-$ character variety $\mathcal{X}(\Sigma)$. This was first noticed by Turaev [Tu91] and the full picture was unraveled by the works of Bullock, Frohman and KaniaBartoszyńska [Bu97, BFK99, BFK98] and Przytycki and Sikora [PS00]. In our context, the corresponding framework will be that of the decorated Teichmüller space and the notion of $\lambda$-lengths, which as we will see can be understood as generalized trace functions.

3.1. The decorated Teichmüller space and its Poisson structure. As before, we let $\Sigma$ be a surface with a nonempty set of punctures $V=\left\{v_{1}, \ldots, v_{s}\right\}$. In order to work in the hyperbolic setting, we suppose in addition that $\chi(\Sigma)<$ 0 . We consider the cusped Teichmüller space $\mathcal{T}_{c}(\Sigma)$ defined as the set of Teichmüller equivalence classes of complete hyperbolic metrics on $\Sigma$ with finite area. We recall that two hyperbolic metrics $m$ and $m^{\prime}$ on $\Sigma$ are called Teichmüller equivalent if there exists an isometry from $(\Sigma, m)$ to $\left(\Sigma, m^{\prime}\right)$ that is isotopic to the identity map of $\Sigma$. A decoration $r \in \mathbb{R}_{>0}^{V}$ is given by a choice of a positive real number $r_{i}=r\left(v_{i}\right)$ associated to each puncture. Geometrically, given a metric $m \in \mathcal{T}_{c}(\Sigma)$, a decoration should be interpreted as a choice of a horocycle of length $r_{i}$ at each puncture $v_{i}$ of $\Sigma$. The decorated Teichmüller space $\mathcal{T}^{d}(\Sigma)$, introduced by Penner in [Pe87], is then defined to be the space of decorated hyperbolic metrics $(m, r), m \in \mathcal{T}_{c}(\Sigma)$. Topologically, it is the fiber bundle $\mathcal{T}^{d}(\Sigma)=\mathcal{T}_{c}(\Sigma) \times \mathbb{R}_{>0}^{V}$ over the cusped Teichmüller space.

One of the reasons for introducing decorations is to be able to measure the length of an arc between punctures. More precisely, Let $\alpha$ be an arc between two punctures of $\Sigma$, possibly with self-intersections. Given a decorated hyperbolic metric $(m, r)$, consider a geodesic lift $\widetilde{\alpha}$ of $\alpha$ to the universal cover $\mathbb{H}^{2}$ of $(\Sigma, m)$. The length $l(\alpha)$ of $\alpha$ for $(m, r)$ is then defined to be the signed 
length of the segment of $\widetilde{\alpha}$ between the horocycles given by the decoration, where the sign is chosen to be positive if the horocycles do not intersect and negative if they do. A number of properties concerning lengths of arcs are in fact best expressed in terms of the associated $\lambda$-length

$$
\lambda(\alpha)=e^{\frac{l(\alpha)}{2}} .
$$

In particular, if $a, b, c$ and $d$ are the consecutive sides of a decorated ideal quadrilateral in $\Sigma$ and $e$ and $e^{\prime}$ are its diagonals, they satisfy the Ptolemy relation [Pe87]

$$
\lambda(e) \lambda\left(e^{\prime}\right)=\lambda(a) \lambda(c)+\lambda(b) \lambda(d) .
$$

Let $T$ be an ideal triangulation of $\Sigma$, that is, a maximal collection of isotopy classes of simple arcs between punctures in $\Sigma$ which decomposes the surface into ideal triangles. We let $E$ be the set of edges of $T$. If $\Sigma$ is a sphere with at least three punctures or a surface of genus $g>0$ with at least one puncture then such a triangulation exists. In this case, the associated lengths $l(e), e \in E$, form a coordinate system on $\mathcal{T}^{d}(\Sigma)$. In these coordinates, Mondello [Mo09] introduced a Poisson bi-vector field on $\mathcal{T}^{d}(\Sigma)$ defined as follows: on a decorated hyperbolic surface $\Sigma$, given an end of an edge $\alpha$ and an end of an edge $\beta$ meeting at a puncture $v$, we define the generalized angle from the end of $\alpha$ to the end of $\beta$ to be the length of the horocycle segment between them going in the positive direction for the orientation of $\Sigma$. We then let $\theta_{v}$ be the sum of the generalized angles from each end of $\alpha$ to each end of $\beta$ meeting at $v$, and we let $\theta_{v}^{\prime}$ be the sum of the generalized angles from the ends of $\beta$ to the ends of $\alpha$ (see the figure below for an example).

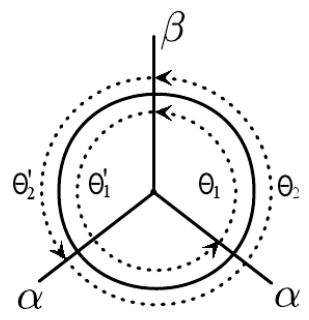

Figure 2. here $\theta_{v}=\theta_{1}+\theta_{2}$ and $\theta_{v}^{\prime}=\theta_{1}^{\prime}+\theta_{2}^{\prime}$.

We then consider the following bi-vector field

$$
\Pi_{W P}=\frac{1}{4} \sum_{v \in V} \sum_{\substack{\alpha, \beta \in E \\ \alpha \cap \beta=v}} \frac{\theta_{v}^{\prime}-\theta_{v}}{r(v)} \frac{\partial}{\partial l(\alpha)} \wedge \frac{\partial}{\partial l(\beta)}
$$

on the decorated Teichmüller space. It is a Poisson bi-vector field which is directly related to the pull-back of the Weil-Petersson symplectic structure on $\mathcal{T}_{c}(\Sigma)$ as described by Penner in [Pe92] (see Prop 4.7 in [Mo09]) and as such we call it the Weil-Petersson Poisson bi-vector on $\mathcal{T}^{d}(\Sigma)$. It can also be 
shown by a direct computation that this bi-vector is invariant under a diagonal exchange, and as a consequence is independent of the choice of the ideal triangulation $T$.

If $\alpha$ is a loop on $\Sigma$ and $m \in \mathcal{T}_{c}(\Sigma)$, we consider the quantity

$$
\lambda(\alpha)=2 \cosh \frac{l(\alpha)}{2}
$$

where $l(\alpha)$ is the length of the geodesic representative of $\alpha$ in $m$. By convention, we set $l(\alpha)$ to be 0 if $\alpha$ is null-homotopic or homotopic to a puncture. Up to a sign, $\lambda(\alpha)$ is equal to the trace $\operatorname{tr}(\rho(\alpha))$ of (a lift of) the monodromy representation $\rho: \pi_{1}(\Sigma) \rightarrow S L_{2}(\mathbb{R})$ associated to $m$. We purposely used the same notations as for $\lambda$-lengths and call $\lambda(\alpha)$ the generalized trace of $\alpha$, where $\alpha$ can be an arc or a loop on $\Sigma$.

The goal of this section is to construct a map from the algebra of curves $\mathcal{C}(\Sigma)$ to the algebra of functions over $\mathcal{T}^{d}(\Sigma)$ by associating to a generalized curve the product of the generalized traces of its components. One issue, however, is the fact that elements of $\mathcal{C}(\Sigma)$ are not identified up to Reidemeister Move I, which leads us to the following definition. We suppose that $\alpha$ consists of one component. For each point of self-intersection $p$ of $\alpha$, one of its two resolutions at $p$ is connected and the other one is not. We call the former the non-separating resolution and the latter the separating resolution. Note that if $\alpha$ is an arc, then a separating resolution consists of an arc and a loop, which we call the arc component and the loop component respectively. Then we say that a point of self-intersection $p$ of a loop or an arc $\alpha$ is of Type I if one of the components of the separating resolution of $\alpha$ at $p$ is null-homotopic. In the case of an arc this can only be the loop component.

Definition 3.1. If $\alpha$ is a non-null-homotopic loop or a non-trivial arc, then the curling number $c(\alpha)$ of $\alpha$ is defined to be the number of Type I points of self-intersection of $\alpha$. If $\alpha$ is a null-homotopic loop, then $c(\alpha)$ is defined to be the same number plus 1 .

Note that we will not need to specify the curling number of a trivial arc.

Example 3.2. By definition $c(\odot)=0$ and $c(\bigcirc)=1$.

Example 3.3. Since a geodesic minimizes self-intersections in its homotopy class, its curling number is necessarily 0 .

The geometric intuition of the curling number is the following. Among the curves in the regular homotopy class of an arc or a loop $\alpha$, there are representatives $\widetilde{\alpha}$ which look like "geodesics with curls" (or a "trivial loop with curls" if $\alpha$ is null-homotopic). That is, all the null-homotopic components of the separating resolutions of $\widetilde{\alpha}$ at the Type I points of self-intersections are embedded trivial loops corresponding to curls in the curve, and after removing them, the remainder is regularly homotopic to a geodesic. In this case, the quantity $c(\widetilde{\alpha})$ measures exactly the number of curls that $\widetilde{\alpha}$ carries. Then, since the number of Type I points of self-intersection is invariant under Reidemeister Moves II' 

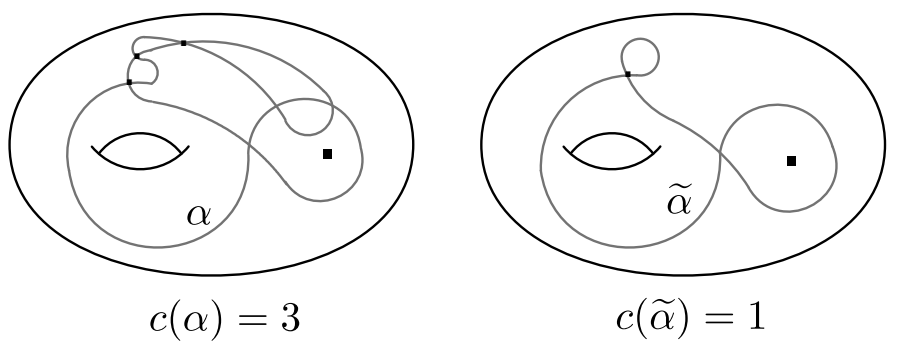

and III, and changes by 0 or \pm 2 under Reidemeister Move II, the curling number $c(\alpha)$ counts the number of curls modulo two, and the quantity $(-1)^{c(\alpha)}$ is well defined for the regular homotopy class of $\alpha$.

Using this definition, if $\alpha=\alpha_{1} \cup \cdots \cup \alpha_{n}$ is a generalized curve, that is, a union of arcs and loops on $\Sigma$, then we let $c(\alpha)=\sum_{i} c\left(\alpha_{i}\right)$ and $\lambda(\alpha)=$ $\prod_{i} \lambda\left(\alpha_{i}\right)$. We recall that, if $(m, r)$ is a decorated hyperbolic metric, then $r(v)$ denotes the length of the horocycle at the puncture $v$ of $\Sigma$. We think of the quantities $r(v)$ and $\lambda(\alpha)$ as functions of the underlying decorated metric and denote by $C^{\infty}\left(\mathcal{T}^{d}(\Sigma)\right)$ the space of $\mathbb{C}$-valued smooth functions on the decorated Teichmüller space $\mathcal{T}^{d}(\Sigma)$.

Theorem 3.4. The map

$$
\Phi: \mathcal{C}(\Sigma) \rightarrow C^{\infty}\left(\mathcal{T}^{d}(\Sigma)\right)
$$

defined on the generators by

$$
\Phi(v)=r(v)
$$

if $v$ is a puncture and

$$
\Phi(\alpha)=(-1)^{c(\alpha)} \lambda(\alpha)
$$

if $\alpha$ is a generalized curve is a well-defined Poisson algebra homomorphism with respect to the Goldman bracket $\{$,$\} on \mathcal{C}(\Sigma)$ and the Weil-Petersson Poisson bracket on $C^{\infty}\left(\mathcal{T}^{d}(\Sigma)\right)$ associated to the bi-vector field $4 \Pi_{W P}$.

The remainder of this article will be dedicated to the proof of this theorem. The first step, done in Section 3.2, will be to derive a series of lengths identities in hyperbolic geometry which generalize the Ptolemy relation, the trace identity and Wolpert's cosine formula for the Weil-Petersson Poisson bracket of length functions. In Section 3.3, Together with an analysis of the behavior of the curling number under resolutions, half of these identities will be combined into generalized trace identities which imply that the map $\Phi$ is an algebra homomorphism. Finally, in Section 3.4, combined with a lemma about the expression of generalized trace functions in terms of the $\lambda$-lengths associated to the edges of a fixed ideal triangulation, the other half will be used to show that this homomorphism respects the Poisson structures. Explicit formulae for the generalized trace in terms of the $\lambda$-lengths associated to the edges of an ideal triangulation are derived in Section 3.5. 
3.2. The lengths identities. In this section we are going to derive a series of identities involving geodesic lengths of curves and arcs between horocycles which are the heart of the proof of Theorem 3.4. They rely on a set of "cosine laws" for various types of generalized hyperbolic triangles which can be found in Appendix A of [GL09]. We will use the results and notations found in their paper throughout this section. Some "twisted versions" of these laws are also needed and are included in Appendix A of this paper. In Lemma 3.5 through 3.8, we study the relationship between the lengths of two intersecting geodesics $\alpha$ and $\beta$, the lengths of their possible resolutions and the (generalized) angle from $\alpha$ to $\beta$. There are several cases depending on whether $\alpha$ or $\beta$ is an arc or a loop and whether the intersection happens inside of $\Sigma$ or at the puncture. Similarly, in Lemma 3.11 through 3.13 we study the relationship between the length of a curve $\alpha$ with a self-intersection and the lengths of its possible resolutions. A complication here comes from the behavior of the curling number of the resolutions of a geodesic curve at a self-intersection. This is treated in Lemma 3.10.

Throughout this section, we will fix a decorated hyperbolic metric $(m, r) \in$ $\mathcal{T}^{d}(\Sigma)$. We recall that if $\alpha$ and $\beta$ are two geodesics on $\Sigma$ for $(m, r)$, then the angle from $\alpha$ to $\beta$ at $p \in \alpha \cap \beta$ in $\Sigma$ is the angle measured from $\alpha$ to $\beta$ for the orientation of $\Sigma$, and the generalized angle from $\alpha$ to $\beta$ at $v \in \alpha \cap \beta, v \in V$ is the length of the horocycle segment measured from $\alpha$ to $\beta$ for the orientation of $\Sigma$. Recall also that, by convention, the length of a loop or an arc that is null-homotopic or homotopic to a puncture is set to be 0 .

We start with the case of two loops intersecting in $\Sigma$. The following formulae are well-known, Part (1) is the trace identity (see for example [Bu97, PS00]) and Part (2) can be interpreted as Wolpert's cosine formula [Wo83] applied to trace functions. We nonetheless give a proof of these formulae for completeness.

Lemma 3.5. Let $\alpha$ and $\beta$ be two closed geodesics of lengths $a$ and $b$, and let $\theta$ be the angle from $\alpha$ to $\beta$ at $p \in \alpha \cap \beta$. If $x$ and $y$ are the lengths of the geodesic representatives of $\alpha_{p} \beta^{+}$and $\alpha_{p} \beta^{-}$respectively, then we have

(1) $\cosh \frac{x}{2}+\cosh \frac{y}{2}=2 \cosh \frac{a}{2} \cosh \frac{b}{2}$,

(2) $\cosh \frac{x}{2}-\cosh \frac{y}{2}=2 \sinh \frac{a}{2} \sinh \frac{b}{2} \cos \theta$.

Proof. We consider Figure 3 (A). Let $\overline{0 \infty}$ be a lift of the geodesic $\beta$ in the universal cover $\mathbb{H}^{2}$ of $\Sigma$. Let $\left\{B_{i}\right\}_{i \in \mathbb{Z}}$ be the lifts of $p$ on $\overline{0 \infty}$ so that $\left|B_{i} B_{i+1}\right|=b$. Let $A_{i}$ and $C_{i}$ for $i=1,2$ be the points on the lift of the geodesic $\alpha$ passing through $B_{i}$ so that $\left|A_{i} B_{i}\right|=\left|B_{i} C_{i}\right|=\frac{a}{2}$, hence $\left|A_{i} C_{i}\right|=$ $a$ and $\overline{A_{i} C_{i}}$ is a lift of $\alpha$ for $i=1,2$. Now take the mid-point $M$ of $\overline{B_{1} B_{2}}$, and connect $A_{1}$ and $C_{2}$ to $M$ by geodesics. Since $\left|A_{1} B_{1}\right|=\left|B_{2} C_{2}\right|=\frac{a}{2}$ and $\left|B_{1} M\right|=\left|M B_{2}\right|=\frac{b}{2}$, and $\angle A_{1} B_{1} M=\angle M B_{2} C_{2}=\pi-\theta$, the triangles $A_{1} B_{1} M$ and $M B_{2} C_{2}$ are isometric, hence the anlges $\angle A_{1} M B_{1}=\angle B_{2} M C_{2}$ and $\angle B_{1} A_{1} M=\angle M C_{2} B_{2}$. Therefore, the points $A_{1}, M$ and $C_{2}$ are on 


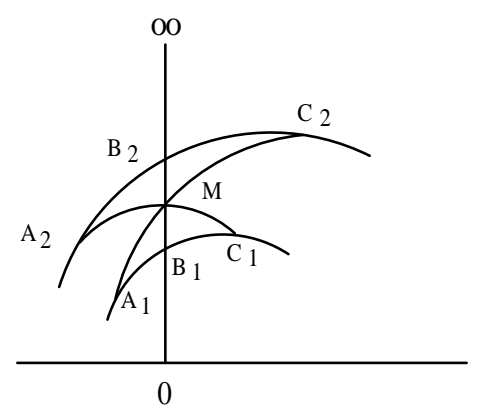

(A)

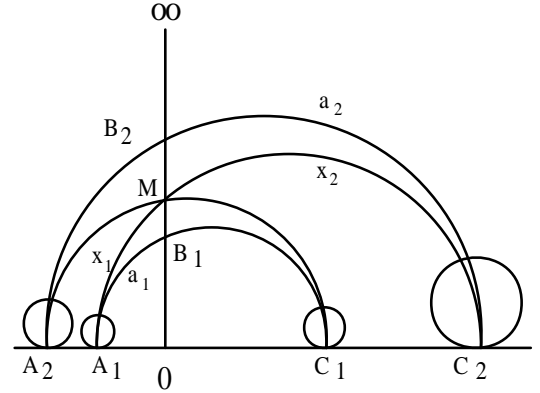

(B)

Figure 3

the geodesic representing a lift of $\alpha_{p} \beta^{+}$, and $\left|A_{1} M\right|=\left|M C_{2}\right|=\frac{x}{2}$. By the same argument, we have that $A_{2}, M$ and $C_{1}$ are on a lift of $\alpha_{p} \beta^{-}$and $\left|A_{2} M\right|=\left|M C_{1}\right|=\frac{y}{2}$. Applying the cosine law to the triangles $A_{1} B_{1} M$ and $A_{2} B_{2} M$ respectively, we have

$$
\begin{aligned}
\cos (\pi-\theta) & =\frac{-\cosh \frac{x}{2}+\cosh \frac{a}{2} \cosh \frac{b}{2}}{\sinh \frac{a}{2} \sinh \frac{b}{2}} \text { and } \\
\cos \theta & =\frac{-\cosh \frac{y}{2}+\cosh \frac{a}{2} \cosh \frac{b}{2}}{\sinh \frac{a}{2} \sinh \frac{b}{2}} .
\end{aligned}
$$

Since $\cos (\pi-\theta)=-\cos \theta$, the sum of the two equalities implies Part (1) and the difference implies Part (2).

q.e.d.

Lemma 3.6. Let $\alpha$ be a geodesic arc of length $a$ and let $\beta$ be a closed geodesic of length $b$. Let $\theta$ be the angle from $\alpha$ to $\beta$ at $p \in \alpha \cap \beta$. If $x$ and $y$ are the lengths of geodesic representatives of the ideal arcs $\alpha_{p} \beta^{+}$and $\alpha_{p} \beta^{-}$ respectively, then we have

(1) $e^{\frac{x}{2}}+e^{\frac{y}{2}}=2 e^{\frac{a}{2}} \cosh \frac{b}{2}$,

(2) $e^{\frac{x}{2}}-e^{\frac{y}{2}}=2 e^{\frac{a}{2}} \sinh \frac{b}{2} \cos \theta$.

Proof. Let us look at Figure 3 (B). Let $\overline{0 \infty}$ be a lift of $\beta$ in the universal cover $\mathbb{H}^{2}$. Let $\left\{B_{i}\right\}_{i \in \mathbb{Z}}$ be the lifts of $p$ on $\overline{0 \infty}$ so that $\left|B_{i}, B_{i+1}\right|=b$, and let $A_{i}$ and $C_{i}$ for $i=1,2$ be the end points of the lifts of $\alpha$ passing through $B_{i}$. Let $M$ be the intersection of $\overline{0 \infty}$ and the geodesic connecting $A_{1}$ and $C_{2}$. Let $a_{1}$ be the distance from $B_{i}$ to the horocycle centered at $A_{i}$ and let $a_{2}$ be the distance from $B_{i}$ to $C_{i}$ for $i=1,2$ so that $a_{1}+a_{2}=a$, and let $x_{1}$ be the distance from $M$ to the horocycle centered at $A_{1}$ and let $x_{2}$ be the distance from $M$ to the horocycle centered at $C_{2}$ so that $x_{1}+x_{2}=x$. Since $\angle A_{1} B_{1} M=\angle C_{2} B_{2} M$ and $\angle A_{1} M B_{1}=\angle C_{2} M B_{2}$, we have that the ideal triangles $A_{1} B_{1} M$ and $C_{2} B_{2} M$ of type $(0,1,1)$ are isometric which implies 
that $\left|B_{1} M\right|=\left|M B_{2}\right|=\frac{b}{2}$. Applying the cosine law to the triangle $A_{1} B_{1} M$, we have

$$
\cos (\pi-\theta)=\frac{-e^{x_{1}}+e^{a_{1}} \cosh \frac{b}{2}}{e^{a_{1}} \sinh \frac{b}{2}} .
$$

Applying the sine law to the triangles $A_{1} B_{1} M$ and $C_{2} B_{2} M$, we have

$$
\frac{e^{a_{1}}}{e^{x_{1}}}=\frac{\sin \angle A_{1} M B_{1}}{\sin \angle A_{1} B_{1} M}=\frac{\sin \angle C_{2} M B_{2}}{\sin \angle C_{2} B_{2} M}=\frac{e^{a_{2}}}{e^{x_{2}}} \text { hence } \frac{a_{2}-a_{1}}{2}=\frac{x_{2}-x_{1}}{2} .
$$

Using this, the cosine law above becomes

$$
\cos (\pi-\theta)=\frac{-e^{\frac{x}{2}}+e^{\frac{a}{2}} \cosh \frac{b}{2}}{e^{\frac{a}{2}} \sinh \frac{b}{2}}
$$

By the same argument applied to the generalized triangles $A_{2} B_{2} M$ and $B_{1} C_{1} M$, we obtain

$$
\cos \theta=\frac{-e^{\frac{y}{2}}+e^{\frac{a}{2}} \cosh \frac{b}{2}}{e^{\frac{a}{2}} \sinh \frac{b}{2}} .
$$

Part (1) is obtained by taking the sum of the two equalities above and Part (2) by taking their difference.

q.e.d.

The following lemma involving $\lambda$-length can be found in [Pe92] (Lemma A1). Part (1) was proved first by Penner in [Pe87] and is the celebrated Ptolemy relation.

Lemma 3.7. (Penner [Pe92]) Let $\alpha$ and $\beta$ be two geodesic arcs of lengths $a$ and $b$, and let $\theta$ be the angle from $\alpha$ to $\beta$ at $p \in \alpha \cap \beta$ in $\Sigma$. If $x$ and $x^{\prime}$ respectively are the lengths of the geodesic representatives of the components of $\alpha_{p} \beta^{+}$, and $y$ and $y^{\prime}$ respectively are the lengths of the geodesic representatives of the components of $\alpha_{p} \beta^{-}$, then we have

(1) $e^{\frac{x}{2}} e^{\frac{x^{\prime}}{2}}+e^{\frac{y}{2}} e^{\frac{y^{\prime}}{2}}=e^{\frac{a}{2}} e^{\frac{b}{2}}$,

(2) $e^{\frac{x}{2}} e^{\frac{x^{\prime}}{2}}-e^{\frac{y}{2}} e^{\frac{y^{\prime}}{2}}=e^{\frac{a}{2}} e^{\frac{b}{2}} \cos \theta$.

Lemma 3.8. Let $\alpha$ and $\beta$ be two geodesic arcs of lengths $a$ and $b$ each having exactly one end at a puncture $v$, and let $\theta$ be the generalized angle from $\alpha$ to $\beta$ and $\theta^{\prime}$ be the generalized angle from $\beta$ to $\alpha$. Let $r$ be the length of the horocycle centered at $v$, and let $x$ and $y$ be the lengths of the geodesic representatives of $\alpha_{v} \beta^{+}$and $\alpha_{v} \beta^{-}$respectively. Then we have

(1) $e^{\frac{x}{2}}+e^{\frac{y}{2}}=r e^{\frac{a}{2}} e^{\frac{b}{2}}$,

(2) $e^{\frac{x}{2}}-e^{\frac{y}{2}}=\left(\theta^{\prime}-\theta\right) e^{\frac{a}{2}} e^{\frac{b}{2}}$.

Proof. Let us look at Figure 4. Let $C$ be a lift of $v$ in the universal cover $\mathbb{H}^{2}$, and let $\overline{A_{1} C}$ and $\overline{B_{1} C}$ be the respective lifts of $\alpha$ and $\beta$ passing through $C$. Then $\overline{A_{2} B_{1}}$ and $\overline{A_{1} B_{1}}$ are lifts of $\alpha_{v} \beta^{+}$and $\alpha_{v} \beta^{-}$, respectively. Applying the cosine law to the ideal triangles $C A_{2} B_{1}$ and $C A_{1} B_{1}$, we have

$$
\theta^{\prime}=e^{\frac{x-a-b}{2}} \text { and } \theta=e^{\frac{y-a-b}{2}} \text {. }
$$




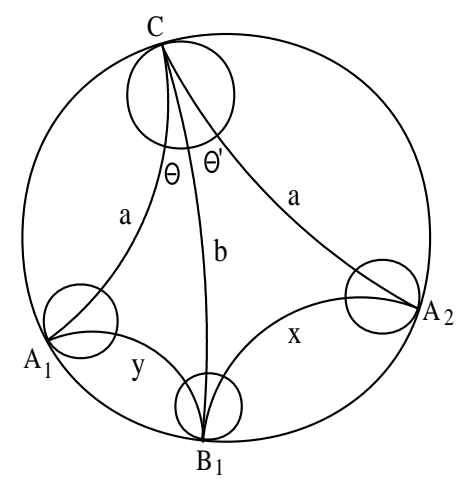

Figure 4

Since $r=\theta+\theta^{\prime}$, the sum of the two equalities above implies part (1), and the difference implies part (2). q.e.d.

Remark 3.9. Note that if $\alpha$ or $\beta$ have several of their ends meeting at $v$, similar formulae hold replacing $x$ and $y$ by all the possible positive and negative resolutions between $\alpha$ and $\beta$, taking the sum of their $\lambda$-lengths instead and considering the sums of the generalized angles between their ends. See the definition of the Goldman bracket on $\mathcal{C}(\Sigma)$ and that of the Weil-Petersson Poisson bi-vector $\Pi_{W P}$ for comparison.

Recall that, for each point of self-intersection $p$ of an arc or a loop $\alpha$, one of its two resolutions at $p$ is connected and the other one is not. The former is called the non-separating resolution of $\alpha$ and the latter is called the separating resolution of $\alpha$. If $\alpha$ is an arc, then the separating resolution of $\alpha$ consists of an arc component and a loop component.

Although the curling number of a geodesic is always 0 , after resolving a self-intersection the curling number of one of its resolutions may be 1 . This happens when $\alpha$ has two points of self-intersections $p$ and $p^{\prime}$ that are connected by two segments of $\alpha$, which, together with a third segment starting and ending at $p$ form an immersed geodesic triangle. The non-separating resolution $\beta$ at $p$ then bounds an immersed disk and the curling number of the resolution is 1 . An example of such a configuration is illustrated in Figure 5 where the triangle and the disk involved are shaded in gray.

Lemma 3.10. Let $\alpha$ be a closed geodesic or a geodesic arc. Then the curling number $c(\beta)$ of the non-separating resolution $\beta$ of $\alpha$ at each of its points of self-intersection is at most 1 and the only possibility that $c(\beta)=1$ is the one described above.

Proof. If $c(\beta)>0$, let $p^{\prime}$ be a Type I self-intersection point of $\beta$ and let $c \subset$ $\beta$ be the loop based at $p^{\prime}$ that is homotopic to the null-homotopic component of the separating resolution of $\beta$ at $p^{\prime}$. Let also $\alpha_{1}$ and $\alpha_{2}$ be the components of $\alpha \backslash p$. We have the following two cases: 


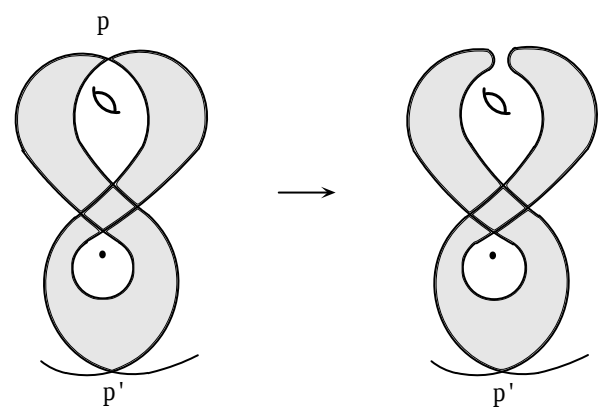

Figure 5. Creation of a curl from a geodesic

(a) $p^{\prime} \in \alpha_{i}$ and $c \subset \alpha_{i}$ for $i=1$ or 2 ,

(b) $p^{\prime} \in \alpha_{i}$ but $c \nsubseteq \alpha_{i}$ for $i=1$ or 2 .

However, if (a) occurred, then $\alpha$ itself would contain a Type I self-intersection point, which is excluded since $\alpha$ is a geodesic. Hence the only possibility is (b), in which case if it is true for say $i=1$, then, necessarily, $\alpha_{2}$ is contained entirely in $c$ and $p^{\prime}$ is the only Type I self-intersection point of $\beta$, otherwise $\alpha$ would also contain one. One can see then that the only possible configuration is the one described above.

q.e.d.

Lemma 3.11. Let $\alpha$ be a closed geodesic of length $a$ and $p$ be one of its self-intersection points. Let $x$ and $y$ respectively be the lengths of the geodesic representatives of the two components of the separating resolution of $\alpha$, and let $z$ be the length of the geodesic representative of the non-separating resolution $\beta$ of $\alpha$.

(1) If $c(\beta)=0$, then

$$
\cosh \frac{a}{2}=2 \cosh \frac{x}{2} \cosh \frac{y}{2}+\cosh \frac{z}{2} .
$$

(2) If $c(\beta)=1$, then

$$
\cosh \frac{a}{2}=2 \cosh \frac{x}{2} \cosh \frac{y}{2}-\cosh \frac{z}{2} .
$$

In addition, the formulae hold when some components of the resolutions of $\alpha$ are circles around a puncture.

Proof. For (1), let us look at (A) of Figure 6. Let $P$ be a lift of $p$ in the universal cover $\mathbb{H}^{2}$, and let $\theta$ the angle between the two lifts $A$ and $B$ of $\alpha$ passing through $P$. Let $X$ and $Y$ be the corresponding lifts of the components of the separating resolution of $\alpha$, and let $Z$ be the corresponding lift of the non-separating resolution of $\alpha$. Let $M_{1} \in A, N_{1} \in B$ and $Z_{1}, Z_{2} \in Z$ be such that $\left|M_{1} Z_{1}\right|$ realizes the distance $d(A, Z)$ and $\left|N_{1} Z_{2}\right|$ realizes the distance $d(B, Z)$. Let $P^{\prime}$ be the lift of $p$ on $A$ next to $P$ and let $B^{\prime}$ be the other lift of $\alpha$ passing through $P^{\prime}$. Let $N_{1}^{\prime} \in B^{\prime}$ and $Z_{2}^{\prime} \in Z$ be such that $\left|N_{1}^{\prime} Z_{2}^{\prime}\right|$ realizes $d\left(B^{\prime}, Z\right)$. Then $\overline{N_{1}^{\prime} Z_{2}^{\prime}}$ and $\overline{N_{1} Z_{2}}$ are the lifts of the same 


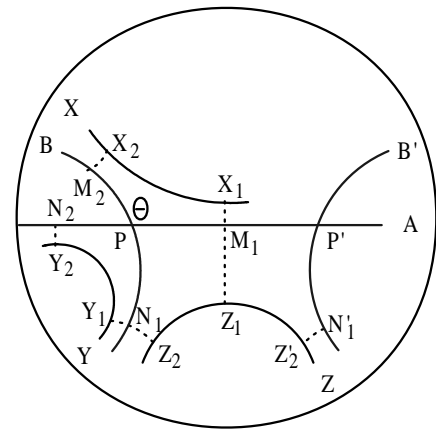

(A)

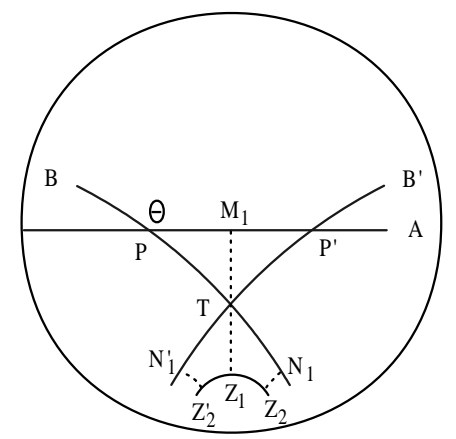

(B)

Figure 6

geodesic segment, which implies that $\left|N_{1}^{\prime} Z_{2}^{\prime}\right|=\left|N_{1} Z_{2}\right|$. Since $\angle N_{1}^{\prime} P^{\prime} M_{1}=$ $\angle N_{1} P M_{1}$, the generalized triangles $M_{1} Z_{1} Z_{2}^{\prime} N_{1}^{\prime} P^{\prime}$ and $M_{1} Z_{1} Z_{2} N_{1} P$ of type $(1,-1,-1)$ are isometric. Therefore, the lengths $\left|Z_{2}^{\prime} Z_{1}\right|=\left|Z_{1} Z_{2}\right|=\frac{z}{2}$ and $\left|P^{\prime} M_{1}\right|=\left|P M_{1}\right| \doteq a_{1}$. Let $M_{1}^{\prime \prime} \in A$ and $X_{1} \in X$ be such that $\left|M_{1}^{\prime \prime} X_{1}\right|$ realizes $d(A, X)$, and let $N_{1}^{\prime \prime} \in B$ and $Y_{1} \in Y$ be such that $\left|N_{1}^{\prime \prime} Y_{1}\right|$ realizes $d(B, Y)$. By a similar argument as above, we see that $\left|P M_{1}^{\prime \prime}\right|=\frac{1}{2}\left|P^{\prime} P\right|=a_{1}$ and $P N_{1}^{\prime \prime}=P N_{1} \doteq a_{2}$. Let $M_{2} \in B, N_{2} \in A, X_{2} \in X$ and $Y_{2} \in Y$ be such that $\left|M_{2} X_{2}\right|$ realizes $d(B, X)$ and $\left|N_{2} Y_{2}\right|$ realizes $d(A, Y)$. Then as above, we have $\left|P M_{2}\right|=a_{1}$ and $\left|P N_{2}\right|=a_{2}$. Since $\left|P M_{1}\right|=\left|P M_{2}\right|=$ $\frac{1}{2}\left|P^{\prime} P\right|$, the points $M_{1}$ and $M_{2}$ cover the same point on the surface $\Sigma$, hence $X_{1}$ and $X_{2}$ cover the same point on $\Sigma$ and $\left|X_{1} X_{2}\right|=x$. The same argument implies $\left|Y_{1} Y_{2}\right|=y$. Applying the cosine law to the generalized triangles $P M_{1} X_{1} X_{2} M_{2}$ and $P N_{1} Y_{1} Y_{2} N_{2}$ of type $(1,-1,-1)$, we have

$$
\cos \theta=\frac{-\cosh x+\sinh ^{2} \frac{a_{1}}{2}}{\cosh ^{2} \frac{a_{1}}{2}}=\frac{-\cosh y+\sinh ^{2} \frac{a_{2}}{2}}{\cosh ^{2} \frac{a_{2}}{2}},
$$

which implies

$$
\sin ^{2} \frac{\theta}{2}=\frac{\cosh \frac{x}{2} \cosh \frac{y}{2}}{\cosh \frac{a_{1}}{2} \cosh \frac{a_{2}}{2}} .
$$

Applying the cosine law to the generalized triangle $P M_{1} Z_{1} Z_{2} N_{1}$ of the same type, we have

$$
\cos (\pi-\theta)=\frac{-\cosh \frac{z}{2}+\sinh \frac{a_{1}}{2} \sinh \frac{a_{2}}{2}}{\cosh \frac{a_{1}}{2} \cosh \frac{a_{2}}{2}} .
$$

From the last two equations and the identity $\cos (\pi-\theta)=2 \sin ^{2} \frac{\theta}{2}-1$ we obtain the result. Note that when some components of the resolutions of $\alpha$ are curves around a puncture, then the corresponding lengths $x, y$ or $z$ are taken to be 0 , and the corresponding generalized triangles become union of generalized 
ideal triangles of type $(0,1,1)$. Applying the cosine law for such triangles we obtain formula (1) in these degenerated cases.

For (2), let us look at (B) of Figure 6. Applying similarly the cosine law to the generalized triangles $P M_{1} X_{1} X_{2} M_{2}$ and $P N_{1} Y_{1} Y_{2} N_{2}$ of type $(1,-1,-1)$, we obtain

$$
\cos \theta=\frac{-\cosh x+\sinh ^{2} \frac{a_{1}}{2}}{\cosh ^{2} \frac{a_{1}}{2}}=\frac{-\cosh y+\sinh ^{2} \frac{a_{2}}{2}}{\cosh ^{2} \frac{a_{2}}{2}},
$$

which implies

$$
\sin ^{2} \frac{\theta}{2}=\frac{\cosh \frac{x}{2} \cosh \frac{y}{2}}{\cosh \frac{a_{1}}{2} \cosh \frac{a_{2}}{2}}
$$

Since $c(\beta)=1$, there is an intersection $T$ between $B$ and $B^{\prime}$ and the generalized triangle $P M_{1} Z_{1} Z_{2} N_{1}$ of type $(1,-1,-1)$ is twisted. Applying the cosine law for such generalized triangle (see Appendix A), we have

$$
\cos (\pi-\theta)=\frac{\cosh \frac{z}{2}+\sinh \frac{a_{1}}{2} \sinh \frac{a_{2}}{2}}{\cosh \frac{a_{1}}{2} \cosh \frac{a_{2}}{2}},
$$

and the identity $\cos (\pi-\theta)=2 \sin ^{2} \frac{\theta}{2}-1$ implies the result.

q.e.d.

Lemma 3.12. Let $\alpha$ be a geodesic arc of length $a$ and $p$ be one of its points of self-intersection. For the separating resolution of $\alpha$, let $x$ and $y$ be the lengths of the geodesic representatives of the loop and of the arc component respectively. We also let $z$ be the length of the geodesic representative of the non-separating resolution $\beta$ of $\alpha$.

(1) If $c(\beta)=0$, then

$$
e^{\frac{a}{2}}=2 \cosh \frac{x}{2} e^{\frac{y}{2}}+e^{\frac{z}{2}}
$$

(2) If $c(\beta)=1$, then

$$
e^{\frac{a}{2}}=2 \cosh \frac{x}{2} e^{\frac{y}{2}}-e^{\frac{z}{2}}
$$

In addition, the formulae hold when the loop component in the separating resolution of $\alpha$ is a circle around a puncture.

Proof. For (1), let $P$ in (A) of Figure 7 be a lift of $p$ in the universal cover $\mathbb{H}^{2}$, and let $A$ and $B$ be the two lifts of $\alpha$ passing through $P$ with $\theta$ the angle between them at $p$. Let the end point $Y$ of $A$ and the end point $Y_{1}$ of $B$ respectively be the lifts of the two end points of $\alpha$ so that $\overline{Y Y_{1}}$ is a lift of the geodesic representative of the arc component of the separating resolution of $\alpha$. Let $X$ be the corresponding lift of the geodesic representative of the loop component of the separating resolution of $\alpha$, and let $D$ and $D_{1}$ be the lifts of the geodesic representative of the non-separating resolution of $\alpha$. We take points $X_{1}$ and $X_{2} \in X, M \in B$ and $N \in A$ such that $\left|M X_{1}\right|$ realizes $d(B, X)$ and $\left|N X_{2}\right|$ realizes $d(A, X)$. Since $\overline{M X_{1}}$ and $\overline{N X_{2}}$ cover the same curve on $\Sigma$, we have $\left|M X_{1}\right|=\left|N X_{2}\right|$. Applying the sine law to the generalized triangle $P M X_{1} X_{2} N$ of type $(-1,-1,1)$, we have $|P M|=|P N| \doteq \frac{a_{3}}{2}$. Suppose 


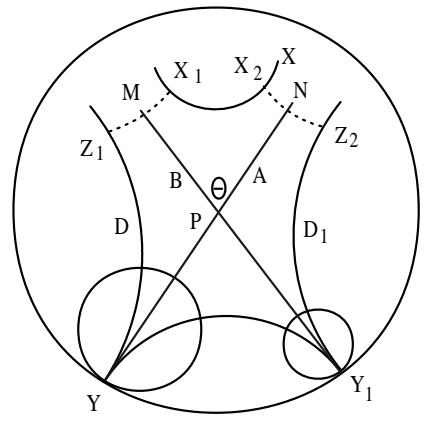

(A)

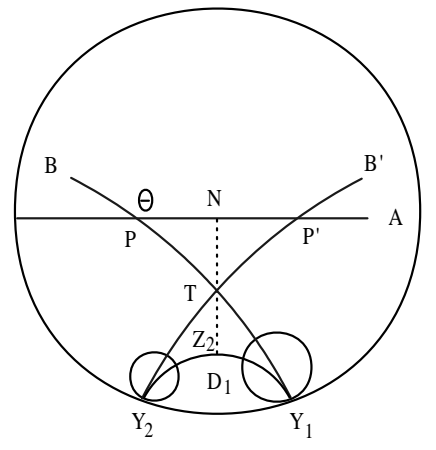

(B)

Figure 7

$M^{\prime} \in A^{\prime}, N^{\prime} \in A, Z_{1} \in D$ and $Z_{2} \in D_{1}$ are the points such that $\left|M^{\prime} Z_{1}\right|$ realizes $d(B, D)$ and $\left|N^{\prime} Z_{2}\right|$ realizes $d\left(A, D_{1}\right)$. Then $\overline{M^{\prime} Z_{1}}$ and $\overline{N^{\prime} Z_{2}}$ cover the same curve on $\Sigma$, hence $\left|M^{\prime} Z_{1}\right|=\left|N^{\prime} Z_{2}\right|$. Applying the cosine law to the generalized ideal triangles $P Y Z_{1} M^{\prime}$ and $P Y_{1} Z_{2} N^{\prime}$ of type $(-1,0,1)$, we see that

$$
\begin{aligned}
\sinh \left|P M^{\prime}\right| & =\frac{1+\cos (\pi-\theta) \cosh \left|M^{\prime} Z_{1}\right|}{\sin (\pi-\theta) \sinh \left|M^{\prime} Z_{1}\right|} \\
& =\frac{1+\cos (\pi-\theta) \cosh \left|N^{\prime} Z_{2}\right|}{\sin (\pi-\theta) \sinh \left|N^{\prime} Z_{2}\right|}=\sinh \left|P N^{\prime}\right| .
\end{aligned}
$$

Therefore we have $\left|P M^{\prime}\right|=\left|P N^{\prime}\right|$. Hence $M^{\prime}=M, N^{\prime}=N$ and $\left|P M^{\prime}\right|=$ $\left|P N^{\prime}\right|=\frac{a_{3}}{2}$. Let $H_{Y}$ and $H_{Y_{1}}$ respectively be the horocycles centered at $Y$ and $Y_{1}$, and $a_{1}=d\left(P, H_{Y}\right), a_{2}=d\left(P, H_{Y_{1}}\right), z_{1}=d\left(Z_{1}, H_{Y}\right)$ and $z_{2}=$ $d\left(Z_{2}, H_{Y_{1}}\right)$. Then $a=a_{1}+a_{2}+a_{3}$ and $z=z_{1}+z_{2}$. Applying the cosine law to the generalized ideal triangle $P Y Z_{1} M$, we have

$$
\cos (\pi-\theta)=\frac{-e^{z_{1}}+e^{a_{1}} \sinh \frac{a_{3}}{2}}{e^{a_{1}} \cosh \frac{a_{3}}{2}} .
$$

From the sine law applied to the generalized ideal triangles $P Y Z_{1} M$ and $P Y_{1} Z_{2} N$, we have

$$
\frac{e^{z_{1}}}{e^{a_{1}}}=\frac{\sin (\pi-\theta)}{\sinh \left|M Z_{1}\right|}=\frac{\sin (\pi-\theta)}{\sinh \left|N Z_{2}\right|}=\frac{e^{z_{2}}}{e^{a_{2}}}, \text { hence } \frac{a_{2}-a_{1}}{2}=\frac{z_{2}-z_{1}}{2} .
$$

Using this, the cosine law above becomes

$$
\cos (\pi-\theta)=\frac{-e^{\frac{z}{2}}+e^{\frac{a_{1}+a_{2}}{2}} \sinh \frac{a_{3}}{2}}{e^{\frac{a_{1}+a_{2}}{2}} \cosh \frac{a_{3}}{2}},
$$

hence

$$
e^{\frac{z}{2}}=e^{\frac{a_{1}+a_{2}}{2}}\left(\sinh \frac{a_{3}}{2}+\cosh \frac{a_{3}}{2} \cos \theta\right) .
$$


Applying the cosine law to the generalized triangle $P M X_{1} X_{2} N$, we have

$$
\cos \theta=\frac{-\cosh x+\sinh ^{2} \frac{a_{3}}{2}}{\cosh ^{2} \frac{a_{3}}{2}}, \text { hence } 2 \cosh \frac{x}{2}=2 \cosh \frac{a_{3}}{2} \sin \frac{\theta}{2},
$$

and the cosine law applied to the generalized ideal triangle $P Y Y_{1}$ of type $(0,0,1)$ gives

Therefore, we have

$$
e^{\frac{y}{2}}=e^{\frac{a_{1}+a_{2}}{2}} \sin \frac{\theta}{2}
$$

$$
\begin{aligned}
2 \cosh \frac{x}{2} e^{\frac{y}{2}}+e^{\frac{z}{2}} & =e^{\frac{a_{1}+a_{2}}{2}}\left(\sinh \frac{a_{3}}{2}+\cosh \frac{a_{3}}{2} \cos \theta+2 \cosh \frac{a_{3}}{2} \sin ^{2} \frac{\theta}{2}\right) \\
& =e^{\frac{a_{1}+a_{2}}{2}} e^{\frac{a_{3}}{2}}=e^{\frac{a}{2}} .
\end{aligned}
$$

For (2), let us look at (B) of Figure 7. Applying similarly the cosine law to the generalized triangle $P M X_{1} X_{2} N$, we have

$$
\cos \theta=\frac{-\cosh x+\sinh ^{2} \frac{a_{3}}{2}}{\cosh ^{2} \frac{a_{3}}{2}}
$$

which implies

$$
2 \cosh \frac{x}{2}=2 \cosh \frac{a_{3}}{2} \sin \frac{\theta}{2},
$$

and the cosine law applied to the generalized ideal triangle $P Y Y^{\prime}$ of type $(0,0,1)$ gives

$$
e^{\frac{y}{2}}=e^{\frac{a_{1}+a_{2}}{2}} \sin \frac{\theta}{2}
$$

When $c(\beta)=1$, there is an intersection $T$ between $A^{\prime}$ and $A^{\prime \prime}$ and the generalized triangles $P N Z_{2} Y^{\prime}$ and $P^{\prime} N Z_{2} Y^{\prime \prime}$ of type $(0,1,-1)$ are twisted. Applying the cosine law to $P N Z_{2} Y^{\prime}$, we have

$$
\cos (\pi-\theta)=\frac{e^{z_{1}}+e^{a_{1}} \sinh \frac{a_{3}}{2}}{e^{a_{1}} \cosh \frac{a_{3}}{2}} .
$$

From the sine law for the generalized ideal triangles $P N Z_{2} Y^{\prime}$ and $P N Z_{2} Y^{\prime \prime}$, we have

$$
\frac{e^{z_{1}}}{e^{a_{1}}}=\frac{\sin (\pi-\theta)}{\sinh \left|N Z_{2}\right|}=\frac{e^{z_{2}}}{e^{a_{2}}}, \text { which implies } \frac{a_{2}-a_{1}}{2}=\frac{z_{2}-z_{1}}{2} .
$$

Using this, the cosine law above becomes

$$
\cos (\pi-\theta)=\frac{e^{\frac{z}{2}}+e^{\frac{a_{1}+a_{2}}{2}} \sinh \frac{a_{3}}{2}}{e^{\frac{a_{1}+a_{2}}{2}} \cosh \frac{a_{3}}{2}}
$$

hence

$$
-e^{\frac{z}{2}}=e^{\frac{a_{1}+a_{2}}{2}}\left(\sinh \frac{a_{3}}{2}+\cosh \frac{a_{3}}{2} \cos \theta\right) .
$$

Therefore, we obtain

$$
2 \cosh \frac{x}{2} e^{\frac{y}{2}}-e^{\frac{z}{2}}=e^{\frac{a}{2}} .
$$


q.e.d.

Lemma 3.13. Let $\alpha$ be a geodesic arc of length a both of whose ends meet at a puncture $v$, and let $r$ be the length of the horocycle centered at $v$. Let also $x$ and $y$ be the lengths of the geodesic representatives of the two resolutions $\alpha_{1}$ and $\alpha_{2}$ of $\alpha$ at $v$. Then we have

$$
e^{\frac{a}{2}}=\frac{2}{r}\left(\cosh \frac{x}{2}+\cosh \frac{y}{2}\right) \text {. }
$$

In addition, the formula holds when some of the components of the resolutions of $\alpha$ are circles around a puncture.

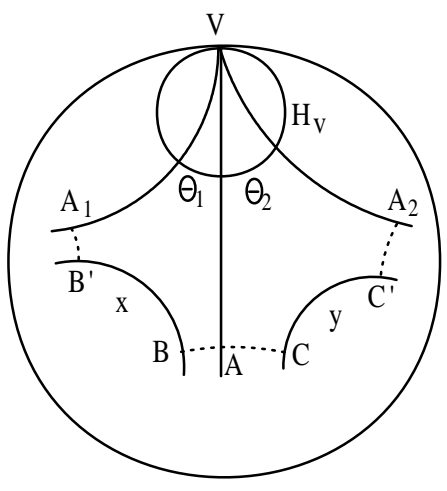

Figure 8

Proof. In Figure 8, let $V$ be the lift of $v$ and let $H_{V}$ be the lift of the horocycle centered at $V$, let $\overline{A V}$ and $\overline{A_{1} V}$ be the lifts of $\alpha$ passing through $V$ in the universal cover $\mathbb{H}^{2}$. Let $\theta_{1}$ be the generalized angle between $\overline{A V}$ and $\overline{A_{1} V}$ and let $\overline{B B^{\prime}}$ be the corresponding lift of the geodesic representative of the homotopy class of $\alpha_{1}$. We take the point $A, A_{1}, B$ and $B^{\prime}$ such that $|A B|$ realizes the distance from $\overline{A V}$ to $\overline{B B^{\prime}}$ and $\left|A_{1} B^{\prime}\right|$ realizes the distance from $\overline{A_{1} V}$ to $\overline{B B^{\prime}}$. Since $\overline{A B}$ and $\overline{A_{1} B^{\prime}}$ cover the same line in $\Sigma$, we have $|A B|=\left|A_{1} B^{\prime}\right|$ and $\left|B B^{\prime}\right|=x$. By the sine law for the generalized triangle $C A B B^{\prime} A^{\prime}$ of type $(0,-1,-1)$, we have

$$
\frac{e^{d\left(A, H_{V}\right)}}{\sinh \left|A_{1} B^{\prime}\right|}=\frac{e^{d\left(A_{1}, H_{V}\right)}}{\sinh |A B|}=1,
$$

which implies that $d\left(A, H_{V}\right)=d\left(A_{1}, H_{V}\right)=\frac{a}{2}$. Applying the cosine law to the generalized triangle $C A B B^{\prime} A^{\prime}$, we have

$$
\theta_{1}^{2}=\frac{\cosh x+1}{\frac{e^{a}}{2}}
$$

which implies that

$$
\theta_{1}=\frac{2 \cosh \frac{x}{2}}{e^{\frac{a}{2}}}
$$


Similarly, if we let $\overline{A_{2} V}$ be the other lift of $\alpha$ adjacent to $\overline{A V}$ and let $\theta_{2}$ be the generalized angle between $\overline{A V}$ and $\overline{A_{2} V}$, we obtain

$$
\theta_{2}=\frac{2 \cosh \frac{y}{2}}{e^{\frac{a}{2}}}
$$

which together with the previous identity implies the formula.

q.e.d.

3.3. Generalized trace identities and the algebra homomorphism. Combining the results from the previous section, we obtain the following generalized trace identities.

Proposition 3.14. (a) For a generalized curve $\alpha$ with $p$ one of its selfintersection points in $\Sigma$, let $\alpha_{1}$ and $\alpha_{2}$ be the components of the separating resolution of $\alpha$ at $p$ and let $\beta$ be the non-separating resolution of $\alpha$ at $p$. Then we have

$$
(-1)^{c(\alpha)} \lambda(\alpha)=(-1)^{c\left(\alpha_{1}\right)+c\left(\alpha_{2}\right)} \lambda\left(\alpha_{1}\right) \lambda\left(\alpha_{2}\right)+(-1)^{c(\beta)} \lambda(\beta) .
$$

(b) For $\alpha$ and $\beta$ two generalized curves with $p \in \Sigma$ one of their intersection points, let $\gamma_{1}$ and $\gamma_{2}$ be the resolutions of $\alpha$ and $\beta$ at $p$. Then we have

$$
(-1)^{c(\alpha)+c(\beta)} \lambda(\alpha) \lambda(\beta)=(-1)^{c\left(\gamma_{1}\right)} \lambda\left(\gamma_{1}\right)+(-1)^{c\left(\gamma_{2}\right)} \lambda\left(\gamma_{2}\right) .
$$

Proof. We use induction on the number of intersection points. If a generalized curve $\alpha$ has only one self-intersection $p$, we have to consider the following two cases:

(1) $p$ is of Type I, and

(2) $p$ is not of Type I.

In case (1), one of $\alpha_{1}$ and $\alpha_{2}$, say $\alpha_{1}$, is a trivial loop. In this case $\lambda\left(\alpha_{1}\right)=2$, $\lambda(\alpha)=\lambda\left(\alpha_{2}\right)=\lambda(\beta), c(\alpha)=c\left(\alpha_{1}\right)=1$ and $c(\beta)=c\left(\alpha_{2}\right)=0$. Then

$$
\begin{aligned}
(-1)^{c\left(\alpha_{1}\right)+c\left(\alpha_{2}\right)} \lambda\left(\alpha_{1}\right) \lambda\left(\alpha_{2}\right)+(-1)^{c(\beta)} \lambda(\beta) & \\
= & -2 \lambda(\alpha)+\lambda(\alpha)=(-1)^{c(\alpha)} \lambda(\alpha) .
\end{aligned}
$$

Hence (a) is true. In case (2), $\alpha$ is regularly homotopic to a geodesic, and (a) is true by Lemma 3.11 and 3.12. If two simple generalized curves $\alpha$ and $\beta$ have only one intersection, then $\alpha$ and $\beta$ are regularly homotopic to geodesics, and (b) is true by (1) of Lemma 3.5 - 3.7.

Now we assume that formula (a) holds when the number of self-intersections of $\alpha$ is less than $n$, and formula (b) holds when the number of crossings of $\alpha \cup \beta$ is less than $n$. We induct on the number of self-intersections of $\alpha$ for (a) and on the number of crossings of $\alpha \cup \beta$ for (b).

For (a), we call a self-intersection $p$ of $\alpha$ of Type II if $p$ is not of Type I and there is a self-intersection $p^{\prime}$ of $\alpha$ such that one of the simultaneous resolutions of $\alpha$ at $p$ and $p^{\prime}$ contains a null-homotopic component. We call such component a (topological) bigon bounded by $\alpha$ with vertices $p$ and $p^{\prime}$. Note that this bigon is not assumed to be immersed. If the number of selfintersections of $\alpha$ is equal to $n$, we have to consider the following three cases: 
(1) $p$ is of Type I,

(2) $p$ is of Type II, and

(3) $p$ is neither of Type I nor of Type II.

In case (1), we have $\lambda(\alpha)=\lambda\left(\alpha_{2}\right)=\lambda(\beta)$ and $c(\alpha)=c\left(\alpha_{1}\right)+c\left(\alpha_{2}\right)=$ $c(\beta)+1$, where the extra Type I self-intersection is given by $p$. As a consequence,

$$
\begin{aligned}
& (-1)^{c\left(\alpha_{1}\right)+c\left(\alpha_{2}\right)} \lambda\left(\alpha_{1}\right) \lambda\left(\alpha_{2}\right)+(-1)^{c(\beta)} \lambda(\beta) \\
& =(-1)^{c(\alpha)} 2 \lambda(\alpha)-(-1)^{c(\alpha)} \lambda(\alpha)=(-1)^{c(\alpha)} \lambda(\alpha) .
\end{aligned}
$$

Hence (a) is true in this case.

In case (2), we let $p^{\prime}$ be the other vertex of the topological bigon $B$. Then $B$ is a component of the separating resolution of one of $\alpha_{1}, \alpha_{2}$ or $\beta$ at $p$. If $B$ is bounded by one of $\alpha_{1}$ and $\alpha_{2}$, say $\alpha_{1}$, then we let $\alpha_{1}^{\prime}$ be the non-nullhomotopic component of the separating resolution of $\alpha_{1}$ at $p^{\prime}$. Note that $\alpha_{1}^{\prime}$ is also one of the components of the separating resolution of $\beta$ at $p^{\prime}$. We let $\alpha_{2}^{\prime}$ be the other component of the separating resolution of $\beta$ at $p^{\prime}$ and let $\alpha^{\prime}$ be the non-separating resolutions of $\beta$ at $p^{\prime}$. Then $\lambda(\alpha)=\lambda\left(\alpha^{\prime}\right), \lambda\left(\alpha_{1}\right)=\lambda\left(\alpha_{1}^{\prime}\right)$, $\lambda\left(\alpha_{2}\right)=\lambda\left(\alpha_{2}^{\prime}\right), c(\alpha)=c\left(\alpha^{\prime}\right)$ and $c\left(\alpha_{1}\right)+c\left(\alpha_{2}\right)=c\left(\alpha_{1}^{\prime}\right)+c\left(\alpha_{2}^{\prime}\right)+1$, where the extra Type I self-intersection is given by $p^{\prime}$. From this, formula (a) is equivalent to

$$
(-1)^{c(\beta)} \lambda(\beta)=(-1)^{c\left(\alpha^{\prime}\right)} \lambda\left(\alpha^{\prime}\right)+(-1)^{c\left(\alpha_{1}^{\prime}\right)+c\left(\alpha_{2}^{\prime}\right)} \lambda\left(\alpha_{1}^{\prime}\right) \lambda\left(\alpha_{2}^{\prime}\right),
$$

which holds by the inductive assumption for (a), since the number of selfintersections of $\beta$ is less than $n$. If $B$ is bounded by $\beta$, we have that one of the resolutions of $\alpha_{1} \cup \alpha_{2}$ at $p^{\prime}$, which we denote by $\alpha^{\prime}$, is homotopic $\alpha$ with $c(\alpha)=c\left(\alpha^{\prime}\right)$. The other resolution of $\alpha_{1} \cup \alpha_{2}$ at $p^{\prime}$, which we denote by $\beta$, is homotopic to $\beta^{\prime}$ with $c(\beta)=c\left(\beta^{\prime}\right)+1$, where the extra Type I self-intersection is given by $p^{\prime}$. Then formula (a) is equivalent to

$$
(-1)^{c\left(\alpha_{1}\right)+c\left(\alpha_{2}\right)} \lambda\left(\alpha_{1}\right) \lambda\left(\alpha_{2}\right)=(-1)^{c\left(\alpha^{\prime}\right)} \lambda\left(\alpha^{\prime}\right)+(-1)^{c\left(\beta^{\prime}\right)} \lambda\left(\beta^{\prime}\right),
$$

which holds by the induction assumption for (b), since the number of crossings of $\alpha_{1} \cup \alpha_{2}$ is less than $n$.

In case (3), we let $\bar{\alpha}$ be the non-null-homotopic component of the separating resolution of $\alpha$ at all of its Type I self-intersections simultaneously. Then $\bar{\alpha}$ is regularly homotopic to the geodesic representative of $\alpha$ and $c(\bar{\alpha})=0$. We let $\bar{\alpha}_{1}$ and $\bar{\alpha}_{2}$ be the components of the separating resolution of $\bar{\alpha}$ and let $\bar{\beta}$ be the non-separating resolution of $\bar{\alpha}$ at $p$. Then $c(\alpha)=c\left(\alpha_{1}\right)+c\left(\alpha_{2}\right)$ and $c(\beta)-c(\alpha)=c(\bar{\beta})-c(\bar{\alpha})=c(\bar{\beta})$. By Lemma 3.11 and 3.12, we have

$$
\begin{aligned}
(-1)^{c(\alpha)} \lambda(\alpha) & =(-1)^{c(\alpha)} \lambda(\bar{\alpha}) \\
& =(-1)^{c(\alpha)}\left(\lambda\left(\overline{\alpha_{1}}\right) \lambda\left(\overline{\alpha_{2}}\right)+(-1)^{c(\bar{\beta})} \lambda(\beta)\right) \\
& =(-1)^{c\left(\alpha_{1}\right)+c\left(\alpha_{2}\right)} \lambda\left(\alpha_{1}\right) \lambda\left(\alpha_{2}\right)+(-1)^{c(\beta)} \lambda(\beta) .
\end{aligned}
$$


For (b), a crossing $p$ of $\alpha \cup \beta$ can also be of Type II if there is a crossing $p^{\prime}$ of $\alpha \cup \beta$ such that one of the simultaneous resolutions bounds is null-homotopic. If the number of crossings of $\alpha \cup \beta$ is equal to $n$, we have to consider the following two cases:

(1) $p$ is of Type II, and

(2) $p$ is not of Type II.

In case (1), we let $p^{\prime}$ be the other vertex of the topological bigon $B$ bounded by $\alpha \cup \beta$. Then $B$ is a component of the separating resolution of one of $\gamma_{1}$ and $\gamma_{2}$, say $\gamma_{1}$, at $p$. We let $\alpha^{\prime}$ be the component of the separating resolution of $\gamma_{2}$ at $p^{\prime}$ that is homotopic to $\alpha$, and let $\beta^{\prime}$ be the component of the separating resolution of $\gamma_{2}$ at $p^{\prime}$ that is homotopic to $\beta$. Then $c(\alpha)+c(\beta)=c\left(\alpha^{\prime}\right)+c\left(\beta^{\prime}\right)$. We let $\gamma_{2}^{\prime}$ be the non-separating resolution of $\gamma_{2}$ at $p^{\prime}$. Then $\gamma_{2}^{\prime}$ is homotopic to $\gamma_{1}$ and $c\left(\gamma_{1}\right)=c\left(\gamma_{2}^{\prime}\right)+1$, where the extra Type I self-intersection is given by $p^{\prime}$. Now formula (b) is equivalent to

$$
(-1)^{c\left(\gamma_{2}\right)} \lambda\left(\gamma_{2}\right)=(-1)^{c\left(\alpha^{\prime}\right)+c\left(\beta^{\prime}\right)} \lambda\left(\alpha^{\prime}\right) \lambda\left(\beta^{\prime}\right)+(-1)^{c\left(\gamma_{2}^{\prime}\right)} \lambda\left(\gamma_{2}^{\prime}\right),
$$

which holds by the induction assumption for formula (a), since the number of self-intersections of $\gamma_{2}$ is less than $n$.

In case (2), we let $\bar{\alpha}$ (resp. $\bar{\beta}$ ) be the non-null-homotopic component of the separating resolution of $\alpha$ (resp. $\beta$ ) at all of its Type I self-intersections, and let $\bar{\gamma}_{1}$ and $\bar{\gamma}_{2}$ be the resolutions of $\bar{\alpha}$ and $\bar{\beta}$ at $p$. Then $c(\alpha)+c(\beta)=c\left(\gamma_{1}\right)=$ $c\left(\gamma_{2}\right)$ and

$$
\begin{aligned}
(-1)^{c(\alpha)+c(\beta)} \lambda(\alpha) \lambda(\beta) & =(-1)^{c(\alpha)+c(\beta)} \lambda(\bar{\alpha}) \lambda(\bar{\beta}) \\
& =(-1)^{c(\alpha)+c(\beta)}\left(\lambda\left(\bar{\gamma}_{1}\right)+\lambda\left(\bar{\gamma}_{2}\right)\right) \\
& =(-1)^{c\left(\gamma_{1}\right)} \lambda\left(\gamma_{1}\right)+(-1)^{c\left(\gamma_{2}\right)} \lambda\left(\gamma_{2}\right) .
\end{aligned}
$$

q.e.d.

Proposition 3.15. (a) For an ideal arc $\alpha$ both of whose ends are at the same puncture $v$, let $\beta$ and $\gamma$ be the resolutions of $\alpha$ at $v$, and let $r(v)$ be the length of the horocycle centered at $v$. Then we have

$$
(-1)^{c(\alpha)} \lambda(\alpha)=\frac{1}{r(v)}\left((-1)^{c(\beta)} \lambda(\beta)+(-1)^{c(\gamma)} \lambda(\gamma)\right) .
$$

(b) For $\alpha$ and $\beta$ two ideal arcs intersecting at a puncture $v$, let $\gamma_{1}$ and $\gamma_{2}$ be the resolutions of $\alpha$ and $\beta$ at $v$. Then we have

$$
(-1)^{c(\alpha)+c(\beta)} \lambda(\alpha) \lambda(\beta)=\frac{1}{r(v)}\left((-1)^{c\left(\gamma_{1}\right)} \lambda\left(\gamma_{1}\right)+(-1)^{c\left(\gamma_{2}\right)} \lambda\left(\gamma_{2}\right)\right) \text {. }
$$

Proof. For (a), we call the puncture $v$ of Type II if there is a self-intersection $p$ of $\alpha$ so that the arc component of the separating resolution of $\alpha$ at $p$ is homotopically trivial. We call this component a topological bigon bounded by $\alpha$ at $v$, and call $p$ its vertex. We have to consider the following two cases:

(1) $v$ is of Type II, and

(2) $v$ is not of Type II. 
For case (1), we let $p$ be the vertex of the topological bigon at $v$, and let $\alpha^{\prime}$ be the non-separating resolution of $\alpha$ at $p$. Then $\lambda(\alpha)=\lambda\left(\alpha^{\prime}\right)$ and $c(\alpha)=$ $c\left(\alpha^{\prime}\right)$. We let $\alpha_{1}$ and $\alpha_{2}$ be the resolutions of $\alpha$ at $v$. Since $p$ is a vertex of a topological bigon at $v, p$ is a Type I self-intersection of one of $\beta$ or $\gamma$, say $\beta$. Then the non-separating resolution of $\beta$ at $p$ is regularly homotopic to one of $\alpha_{1}$ and $\alpha_{2}$, say $\alpha_{1}$, and $\beta$ is homotopic to $\alpha_{1}$. We have $\lambda(\beta)=\lambda\left(\alpha_{1}\right)$ and $c(\beta)=c\left(\alpha_{1}\right)+1$, where the extra Type I self-intersection is given by $p$. The non-separating resolution of $\gamma$ at $p$ is regularly homotopic to $\alpha_{2}$ and one of the components of the separating resolutions of $\gamma$ at $p$ is homotopic to a circle around $v$. We denote by $\gamma_{2}$ this component and by $\gamma_{1}$ the other. Then $\gamma_{1}$ is homotopic to $\alpha_{1}$. We have $\lambda\left(\gamma_{1}\right)=\lambda\left(\alpha_{1}\right)$ and $c\left(\gamma_{1}\right)+c\left(\gamma_{2}\right)=c\left(\alpha_{1}\right)$. By Lemma 3.14, we have

$$
\begin{aligned}
(-1)^{c(\gamma)} \lambda(\gamma) & =(-1)^{c\left(\gamma_{1}\right)+c\left(\gamma_{2}\right)} \lambda\left(\gamma_{1}\right) \lambda\left(\gamma_{2}\right)+(-1)^{c\left(\alpha_{2}\right)} \lambda\left(\alpha_{2}\right) \\
& =(-1)^{c\left(\alpha_{1}\right)} 2 \lambda\left(\alpha_{1}\right)+(-1)^{c\left(\alpha_{2}\right)} \lambda\left(\alpha_{2}\right) .
\end{aligned}
$$

Therefore,

$$
(-1)^{c(\beta)} \lambda(\beta)+(-1)^{c(\gamma)} \lambda(\gamma)=(-1)^{c\left(\alpha_{1}\right)} \lambda\left(\alpha_{1}\right)+(-1)^{c\left(\alpha_{2}\right)} \lambda\left(\alpha_{2}\right) .
$$

In order to prove formula (a), we use induction on the number of self-intersections of $\alpha$ in $\Sigma$. If $\alpha$ has only one self-intersection $p$, then $\alpha^{\prime}$ is regularly homotopic to a geodesic. By Lemma 3.13, we have

$$
\lambda(\alpha)=\lambda\left(\alpha^{\prime}\right)=\frac{1}{r(v)}\left(\lambda\left(\alpha_{1}\right)+\lambda\left(\alpha_{2}\right)\right),
$$

which, together with the previous calculation, gives formula (a). We now assume that formula (a) is true when the number of self-intersections of an arc is less than $n$. If $\alpha$ has $n$ self-intersections, then $\alpha^{\prime}$ has less than $n$ selfintersections, and

$$
\begin{aligned}
(-1)^{c(\alpha)} \lambda(\alpha) & =(-1)^{c\left(\alpha^{\prime}\right)} \lambda\left(\alpha^{\prime}\right) \\
& =\frac{1}{r(v)}\left((-1)^{c\left(\alpha_{1}\right)} \lambda\left(\alpha_{1}\right)+(-1)^{c\left(\alpha_{2}\right)} \lambda\left(\alpha_{2}\right)\right) .
\end{aligned}
$$

From this and the previous calculation we obtain formula (a). In case (2), we have $c(\alpha)=c(\beta)=c(\gamma)$ and the formula follows from the case when $\alpha$ is regularly homotopic to a geodesic.

Formula (b) is a consequence of Lemma 3.8(1); and the proof is similar to that of (a).

q.e.d.

Combining Propositions 3.14 and 3.15, we obtain the following intermediate theorem.

Theorem 3.16. The map $\Phi: \mathcal{C}(\Sigma) \rightarrow C^{\infty}\left(\mathcal{T}^{d}(\Sigma)\right)$ defined in Theorem 3.4 is a well-defined commutative algebra homomorphism.

In [Bu97], Bullock conjectured that the map he constructed from the nonquantum skein algebra to the coordinate ring $\mathcal{X}(S)$ of the character variety 
was in fact an isomorphism, which he reduced to the question of showing that there are no non-zero nilpotent elements in $\mathcal{X}(S)$. This question was later addressed by Przytycki and Sikora [PS00] (see also [CM09] for a direct proof of injectivity). We thus state the following conjecture.

Conjecture 3.17. The map $\Phi: \mathcal{C}(\Sigma) \rightarrow C^{\infty}\left(\mathcal{T}^{d}(\Sigma)\right)$ is injective.

3.4. The homomorphism of Poisson algebras. To complete the proof of Theorem 3.4, we need the following lemma.

Lemma 3.18. Let $T$ be an ideal triangulation of a punctured surface $\Sigma$, and $E$ be its set of edges. Suppose $\alpha$ is a generalized curve on $\Sigma$ and $i(\alpha, e)$ is the number of intersection points of $\alpha$ and $e \in E$. Then the product $\alpha$. $\prod_{e \in E} e^{i(\alpha, e)}$ in $\mathcal{C}(\Sigma)$ can be expressed as a polynomial $P_{\alpha}$ with variables in E.

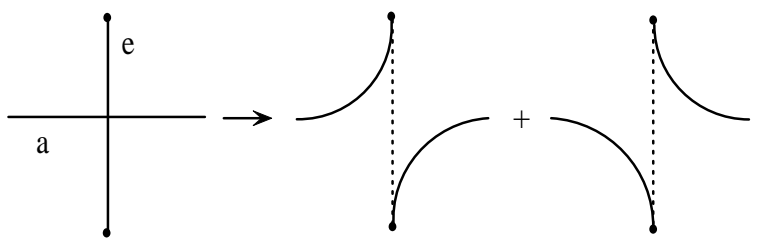

(A)

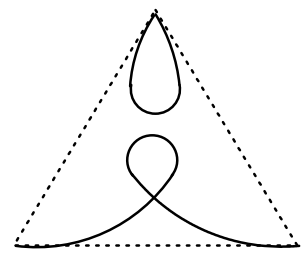

(B)

Figure 9

Proof. Let $e \in E$ such that $\alpha \cap e \neq \emptyset$ and $p \in \alpha \cap e$. As in (A) of Figure 9, each resolution of $\alpha \cdot e$ at $p$ has less intersection number with $e$ than $\alpha$ does. Resolving the product $\alpha \prod_{e \in E} e^{i(\alpha, e)}$ at each point of intersection $p \in \alpha \cap\left(\bigcup_{e \in E} e\right) \cap \Sigma$, we see that each component of the final resolution has no intersection with each edge $e \in E$ in the surface, hence must lie in a triangle in $T$. Since a triangle is contractible, each component of the final resolution is either 0 or up to sign an edge $e \in E$ (as in (B) of Figure 9). q.e.d.

As a direct consequence of Theorem 3.16 and Lemma 3.18, we have the following

Proposition 3.19. Let $T$ be an ideal triangulation of a punctured surface $\Sigma$ endowed with a decorated hyperbolic metric, and $E$ be its set of edges. Then the $\lambda$-length $\lambda(\alpha)$ of any generalized curve $\alpha$ on $\Sigma$ is a Laurent polynomial in $\{\lambda(e) \mid e \in E\}$.

Remark 3.20. In Theorem 3.22 in the next section we give explicit formulae for the generalized trace $\lambda(\alpha)$ in terms of the $\lambda$-lengths associated to the edges of an ideal triangulation, $\alpha$ being either a loop or an ideal arc. 
Proof of Theorem 3.4. For the Poisson structures, we let $T$ be a triangulation of $\Sigma$ with a set of edges $E$. If $e$ and $e^{\prime}$ are two edges in $E$ each having exactly one end meeting at a puncture $v$, and $x$ and $y$ are the resolutions of $e$ and $e^{\prime}$ at $v$, then $\left\{e, e^{\prime}\right\}=\frac{1}{4} v^{-1}(x-y)$. By Theorem 3.16 and Lemma 3.8 (2), we have $\Phi\left(\left\{e, e^{\prime}\right\}\right)=\frac{1}{4 r(v)}\left(e^{\frac{l(x)}{2}}-e^{\frac{l(y)}{2}}\right)=\frac{1}{4} \frac{\theta^{\prime}-\theta}{r(v)} e^{\frac{l(e)}{2}} e^{\frac{l\left(e^{\prime}\right)}{2}}$. We also have that $\Pi_{W P}\left(e^{\frac{l(e)}{2}}, e^{\frac{l\left(e^{\prime}\right)}{2}}\right)=\frac{1}{16} \frac{\theta^{\prime}-\theta}{r(v)} e^{\frac{l(e)}{2}} e^{\frac{l\left(e^{\prime}\right)}{2}}$. If either edge has more than one end meeting at $v$ the same computation holds, replacing $x$ and $y$ by the corresponding sums of resolutions and taking the sums of their $\lambda$-lengths instead (see Remark 3.9 following Lemma 3.8). If $e$ and $e^{\prime}$ are two disjoint edges in $E$, then $\Phi\left(\left\{e, e^{\prime}\right\}\right)=4 \Pi_{W P}\left(e^{\frac{l(e)}{2}}, e^{\frac{l\left(e^{\prime}\right)}{2}}\right)=0$. Therefore, we have that $\Phi\left(\left\{e, e^{\prime}\right\}\right)=4 \Pi_{W P}\left(\lambda(e), \lambda\left(e^{\prime}\right)\right)$ for all pairs of edges in $E$. Now for each generalized curve $\alpha$, by lemma 3.18, we have $\alpha \prod_{e \in E} e^{i(\alpha, e)}=P_{\alpha}$ for some polynomial $P_{\alpha}$ with variables in $E$. Since $\{$,$\} is a Poisson bracket, we have$ $\left\{\alpha \prod_{e \in E} e^{i(\alpha, e)}, e_{0}\right\}=\prod_{e \in E} e^{i(\alpha, e)}\left\{\alpha, e_{0}\right\}+\sum_{e^{\prime} \in E} \alpha \prod_{e \neq e^{\prime}} e^{i(\alpha, e)}\left\{e^{\prime}, e_{0}\right\}$ for each edge $e_{0}$ in $E$, from which we see that $\prod_{e \in E} e^{i(\alpha, e)}\left\{\alpha, e_{0}\right\}=$ $\left\{P_{\alpha}, e_{0}\right\}-Q$ for some polynomial $Q$ in $\alpha, e$ and $\left\{e, e_{0}\right\}$ in which the degrees of $\alpha$ and $\left\{e, e_{0}\right\}$ are equal to 1 . Since $\Phi$ is a $\mathbb{C}$-algebra homomorphism and $\Pi_{W P}$ is a bi-vector field, we have

$$
P_{\alpha}(\lambda(e))=(-1)^{c(\alpha)} \lambda(\alpha) \prod_{e \in E} \lambda(e)^{i(\alpha, e)},
$$

and

$$
\begin{aligned}
& \Pi_{W P}\left((-1)^{c(\alpha)} \lambda(\alpha) \prod_{e \in E} \lambda(e)^{i(\alpha, e)}, \lambda\left(e_{0}\right)\right) \\
= & \prod_{e \in E} \lambda(e)^{i(\alpha, e)} \Pi_{W P}\left((-1)^{c(\alpha)} \lambda(\alpha), \lambda\left(e_{0}\right)\right)+(-1)^{c(\alpha)} Q_{\lambda},
\end{aligned}
$$

where $Q_{\lambda}$ is the value of $Q$ at $\lambda(\alpha), \lambda(e)$ and $\Pi_{W P}\left(\lambda(e), \lambda\left(e_{0}\right)\right)$. As a consequence, since $\lambda(e) \neq 0$ for each $e \in E$, we have

$$
\begin{aligned}
\Phi\left(\left\{\alpha, e_{0}\right\}\right) & =\frac{\Phi\left(\left\{P_{\alpha}, e_{0}\right\}\right)-\Phi(Q)}{\prod_{e \in E} \Phi(e)^{i(\alpha, e)}} \\
& =\frac{4 \Pi_{W P}\left(P_{\alpha}(\lambda(e)), \lambda\left(e_{0}\right)\right)-(-1)^{c(\alpha)} 4 Q_{\lambda}}{\prod_{e \in E} \lambda(e)^{i(\alpha, e)}} \\
& =4 \Pi_{W P}\left((-1)^{c(\alpha)} \lambda(\alpha), \lambda\left(e_{0}\right)\right) .
\end{aligned}
$$

For two generalized curves $\alpha$ an $\beta$, we let $\alpha \prod_{e \in E} e^{i(\alpha, e)}=P_{\alpha}$ and $\beta \prod_{e \in E} e^{i(\beta, e)}=P_{\beta}$ as in Lemma 3.18. Then we have

$$
\prod_{e \in E} e^{i(\alpha, e)+i(\beta, e)}\{\alpha, \beta\}=\left\{P_{\alpha}, P_{\beta}\right\}-R,
$$

where $R$ is a polynomial in $\alpha, \beta, e,\{\alpha, e\},\{e, \beta\}$ and $\left\{e, e^{\prime}\right\}$ such that the degrees of $\alpha, \beta,\{\alpha, e\},\{e, \beta\}$ and $\left\{e, e^{\prime}\right\}$ are all equal to 1 . Therefore, we have 
$\Phi(R)=4 R_{\lambda}$, where $R_{\lambda}$ is the value of $R$ at $\lambda(\alpha), \lambda(\beta), \lambda(e), \Pi_{W P}(\lambda(\alpha)$, $\lambda(e)), \Pi_{W P}(\lambda(e), \lambda(\beta))$ and $\Pi_{W P}\left(\lambda(e), \lambda\left(e^{\prime}\right)\right)$, and

$$
\begin{aligned}
\Phi(\{\alpha, \beta\}) & =\frac{\Phi\left(\left\{P_{\alpha}, P_{\beta}\right\}\right)-\Phi(R)}{\prod_{e \in E} \Phi(e)^{i(\alpha, e)+i(\beta, e)}} \\
& =\frac{4 \Pi_{W P}\left(P_{\alpha}(\lambda(e)), P_{\beta}(\lambda(e))\right)-(-1)^{c(\alpha)+c(\beta)} 4 R_{\lambda}}{\prod_{e \in E} \lambda(e)^{i(\alpha, e)+i(\beta, e)}} \\
& =4 \Pi_{W P}\left((-1)^{c(\alpha)} \lambda(\alpha),(-1)^{c(\beta)} \lambda(\beta)\right) .
\end{aligned}
$$

Let $\pi: \mathcal{T}^{d}(\Sigma) \rightarrow \mathbb{R}_{>0}^{V}$ be the projection onto the fiber. By Mondello [Mo09], the kernel of $\Pi_{W P}$ is the pull-back $\pi^{*}\left(T^{*} \mathbb{R}_{>0}^{V}\right)$ of the cotangent space of $\mathbb{R}_{>0}^{V}$. Since $d(r(v))=\pi^{*}(d v) \in \pi^{*}\left(T^{*} \mathbb{R}_{>0}^{V}\right)$, we have

$$
\Phi(\{v, \alpha\})=4 \Pi_{W P}\left(r(v),(-1)^{c(\alpha)} \lambda(\alpha)\right)=0
$$

for each puncture $v$ and each generalized curve $\alpha$.

q.e.d.

As a consequence of Theorem 3.4, Wolpert's cosine formula generalizes to the bi-vector field $\Pi_{W P}$ as follows:

Corollary 3.21. Let $\theta_{p}$ be the angle from $\alpha$ to $\beta$ at $p \in \alpha \cap \beta$ in $\Sigma$. If $\alpha$ and $\beta$ are two geodesic arcs, then let $\theta_{v}$ be the generalized angle from $\alpha$ to $\beta$ and let $\theta_{v}^{\prime}$ be the generalized angle from $\beta$ to $\alpha$ at a puncture $v \in \alpha \cap \beta$. We have

$$
\Pi_{W P}(l(\alpha), l(\beta))=\frac{1}{2} \sum_{p \in \alpha \cap \beta \cap \Sigma} \cos \theta_{p}+\frac{1}{4} \sum_{v \in \alpha \cap \beta \cap V} \frac{\theta_{v}^{\prime}-\theta_{v}}{r(v)} .
$$

Proof. We let $\lambda^{\prime}(\alpha)=\sinh \frac{l(\alpha)}{2}$ if $\alpha$ is a loop on $\Sigma$, and let $\lambda^{\prime}(\alpha)=\frac{1}{2} e^{\frac{l(\alpha)}{2}}$ if $\alpha$ is an ideal arc on $\Sigma$. By Theorem 3.4 and (2) of Lemma 3.5-3.8, we have

$$
\begin{aligned}
& \Pi_{W P}(l(\alpha), l(\beta)) \\
= & \frac{1}{\lambda^{\prime}(\alpha) \lambda^{\prime}(\beta)} \Pi_{W P}(\lambda(\alpha), \lambda(\beta)) \\
= & \frac{1}{4 \lambda^{\prime}(\alpha) \lambda^{\prime}(\beta)} \Phi(\{\alpha, \beta\}) \\
= & \frac{1}{4 \lambda^{\prime}(\alpha) \lambda^{\prime}(\beta)} \Phi\left(\frac{1}{2} \sum_{p \in \alpha \cap \beta \cap \Sigma}\left(\alpha_{p} \beta^{+}-\alpha_{p} \beta^{-}\right)\right. \\
& \left.\quad+\frac{1}{4} \sum_{v \in \alpha \cap \beta \cap V} \frac{1}{v}\left(\alpha_{v} \beta^{+}-\alpha_{v} \beta^{-}\right)\right)
\end{aligned}
$$




$$
\begin{aligned}
= & \frac{1}{8} \sum_{p \in \alpha \cap \beta \cap \Sigma} \frac{\lambda\left(\alpha_{p} \beta^{+}\right)-\lambda\left(\alpha_{p} \beta^{-}\right)}{\lambda^{\prime}(\alpha) \lambda^{\prime}(\beta)} \\
& +\frac{1}{16} \sum_{v \in \alpha \cap \beta \cap V} \frac{1}{r(v)} \frac{\lambda\left(\alpha_{v} \beta^{+}\right)-\lambda\left(\alpha_{v} \beta^{-}\right)}{\lambda^{\prime}(\alpha) \lambda^{\prime}(\beta)} \\
= & \frac{1}{2} \sum_{p \in \alpha \cap \beta \cap \Sigma} \cos \theta_{p}+\frac{1}{4} \sum_{v \in \alpha \cap \beta \cap V} \frac{\theta_{v}^{\prime}-\theta_{v}}{r(v)} .
\end{aligned}
$$

where if $\alpha_{v} \beta^{ \pm}$corresponds to a sum of resolutions seen as an element in $\mathcal{C}(\Sigma)$, then $\lambda\left(\alpha_{v} \beta^{ \pm}\right)$consists of the sum of their $\lambda$-lengths. $\quad$ q.e.d.

3.5. Trace functions and $\lambda$-lengths: an explicit formula. Let $\Sigma$ be a surface with at least one puncture and such that $\chi(\Sigma)<0$, and choose a decorated hyperbolic metric $(m, r)$ on $\Sigma$. Fix an ideal triangulation $T$ of $\Sigma$ with triangles $\left\{\Delta_{1}, \ldots, \Delta_{s}\right\}$ and edges $\left\{e_{1}, \ldots, e_{t}\right\}$. We let $\alpha$ be a loop or an ideal arc on $\Sigma$ and let $\bar{\alpha}$ be the unique geodesic homotopic to $\alpha$ for the given metric. In addition, we choose an arbitrary orientation for $\bar{\alpha}$. If $\bar{\alpha}$ is a loop, we list the successive triangles that $\bar{\alpha}$ crosses by $\Delta_{i_{1}}$ through $\Delta_{i_{n}}$ starting at some arbitrary point inside the first triangle. Similarly we list the edges that $\bar{\alpha}$ crosses $e_{j_{1}}$ through $e_{j_{n}}$ in such a way that $e_{j_{k}}$ is shared by $\Delta_{i_{k}}$ and $\Delta_{i_{k+1}}$ for $1 \leqslant k \leqslant n-1$ and $e_{j_{n}}$ is shared by $\Delta_{i_{n}}$ and $\Delta_{i_{1}}$. If $\bar{\alpha}$ is an ideal arc, we list triangles and edges accordingly, from Delt $_{i_{1}}$ containing the starting vertex to $\Delta_{i_{n+1}}$ containing the end vertex. Note that in either case the same triangles and edges can occur several times. We denote by $\lambda_{i}=\lambda\left(e_{i}\right)$ the $\lambda$-length of each edge for $(m, r)$.

If $\bar{\alpha}$ makes a left turn in $\Delta_{i_{k}}$ as in (l) of Figure 10, then we let

$$
M_{k}=\left(\begin{array}{cc}
\lambda_{b} & \lambda_{r} \\
0 & \lambda_{l}
\end{array}\right)
$$

for the corresponding labels $b, l$ and $r$ and if $\bar{\alpha}$ makes a right turn in $\Delta_{i_{k}}$ as in (r) of Figure 10, then we let

$$
M_{k}=\left(\begin{array}{cc}
\lambda_{r} & 0 \\
\lambda_{l} & \lambda_{b}
\end{array}\right)
$$

In addition, If $\alpha$ is an ideal arc starting at $\Delta_{i_{1}}$ and ending at $\Delta_{i_{n+1}}$ as in (s) and $(t)$ of Figure 10, then we let

$$
M_{1}=\left(\begin{array}{ll}
\lambda_{r} & \lambda_{l}
\end{array}\right) \quad \text { and } \quad M_{n+1}=\left(\begin{array}{c}
\lambda_{r} \\
\lambda_{l}
\end{array}\right) .
$$

Theorem 3.22. Let $\alpha$ be a generalized curve on a decorated hyperbolic surface $\Sigma$ with a given ideal triangulation $T$, and let $\lambda_{j_{k}}$ and $M_{k}$ be as above. 


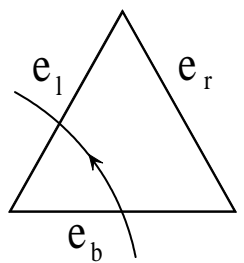

(1)

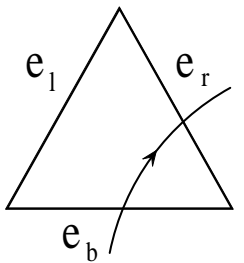

(r)

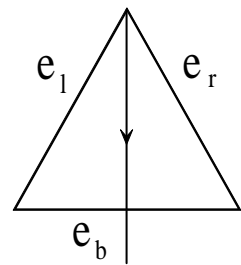

(s)

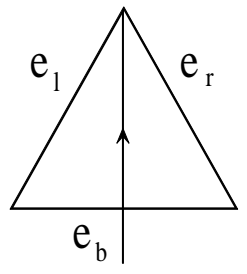

$(\mathrm{t})$

Figure 10. intersections of $\bar{\alpha}$ with ideal triangles

Then we have

$$
\lambda(\alpha)=\left\{\begin{array}{cl}
\frac{\operatorname{tr}\left(M_{1} \cdots M_{n}\right)}{\lambda_{j_{1}} \cdots \lambda_{j_{n}}}, & \text { if } \alpha \text { is a loop, } \\
\frac{M_{1} \cdots M_{n+1}}{\lambda_{j_{1}} \cdots \lambda_{j_{n}}}, & \text { if } \alpha \text { is an ideal arc. }
\end{array}\right.
$$

Proof. We consider the product $\bar{\alpha} \cdot \prod_{k} e_{j_{k}}$ and consider the set $\mathcal{S}$ of the states obtained by simultaneously resolving all the intersections of the product in $\Sigma$. By Theorem 3.16, we have

$$
\lambda(\alpha) \cdot \prod_{k} \lambda_{j_{k}}=\sum_{s \in \mathcal{S}} \lambda(s) .
$$

For each simultaneous resolution $s$ of the product $\bar{\alpha} \cdot \prod_{k} e_{j_{k}}$, each triangle $\Delta_{i_{k}}$ that $\bar{\alpha}$ crosses, starts from or ends at contributes exactly one factor to the lambda lengths $\lambda(s)$ as follows.

If $\bar{\alpha}$ makes a left turn in $\Delta_{i_{k}}$ as in $(l)$ of Figure 10, we have the following four cases according to the type of resolution at the intersections $p_{1}$ and $p_{2}$ of $\bar{\alpha}$ with the edges of $\Delta_{i_{k}}$ :

(11) If $s$ is positive at both $p_{1}$ and $p_{2}$, then there is an ideal arc $\alpha_{++}$in the state which is isotopic to $e_{b}$ as in (l1) of Figure 11. In this case $\Delta_{i_{k}}$ contributes a factor $\lambda_{b}$ to $\lambda(s)$;

(12) If $s$ is positive at $p_{1}$ and negative at $p_{2}$, then there is an ideal arc $\alpha_{+-}$in the state which is isotopic to $e_{r}$ as in (l2) of Figure 11. In this case $\Delta_{i_{k}}$ contributes a factor $\lambda_{r}$ to $\lambda(s)$;

(13) If $s$ is negative at $p_{1}$ and positive at $p_{2}$, then there is an ideal arc $\alpha_{-+}$ in the state which is homotopically trivial as in (l3) of Figure 11. By Lemma $2.12(2), \Delta_{i_{k}}$ contributes a factor 0 to $\lambda(s)$ in this case;

(14) If $s$ is negative at both $p_{1}$ and $p_{2}$, then there is an ideal $\operatorname{arc} \alpha_{--}$in the state which is isotopic to $e_{l}$ as in (l4) of Figure 11. In this case $\Delta_{i_{j}}$ contributes a factor $\lambda_{l}$ to $\lambda(s)$.

If $\bar{\alpha}$ makes a right turn in $\Delta_{i_{k}}$ as in $(r)$ of Figure 10, we similarly have four cases:

(r1) If $s$ is positive at both $p_{1}$ and $p_{2}$, then $\Delta_{i_{k}}$ contributes a factor $\lambda_{r}$ to $\lambda(s)$; 


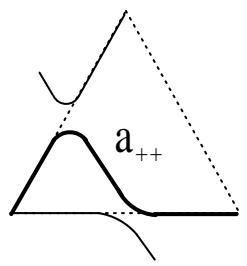

(11)

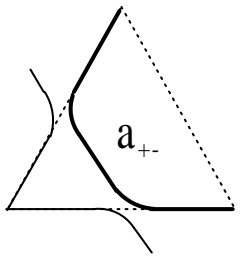

(12)

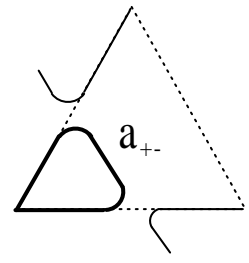

(13)

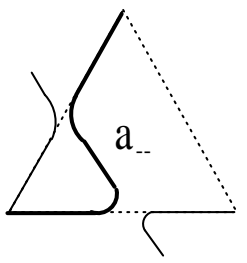

(14)

Figure 11

(r2) If $s$ is positive at $p_{1}$ and negative at $p_{2}$, then $\Delta_{i_{k}}$ contributes a factor 0 to $\lambda(s)$;

(r3) If $s$ is negative at $p_{1}$ and positive at $p_{2}$, then $\Delta_{i_{k}}$ contributes a factor $\lambda_{l}$ to $\lambda(s)$;

(r4) If $s$ is negative at both $p_{1}$ and $p_{2}$, then $\Delta_{i_{k}}$ contributes a factor $\lambda_{b}$ to $\lambda(s)$.

One can then check that, if $\alpha$ is a loop on $\Sigma$, each simultaneous resolution $s$ of $\bar{\alpha} \cdot \prod_{k} e_{j_{k}}$ corresponds to a summand in the trace of the product matrix $M_{1} \cdots M_{n}$, and by the above discussion the lambda lengths $\lambda(s)$ is equal to the corresponding summand in $\operatorname{tr}\left(M_{1} \cdots M_{n}\right)$. Therefore, we have $\sum_{s \in \mathcal{S}} \lambda(s)=\operatorname{tr}\left(M_{1} \cdots M_{n}\right)$. As a consequence,

$$
\lambda(\alpha)=\frac{\operatorname{tr}\left(M_{1} \cdots M_{n}\right)}{\lambda_{j_{1}} \cdots \lambda_{j_{n}}} .
$$

If $\alpha$ is an ideal arc, the triangles $\Delta_{i_{1}}$ and $\Delta_{i_{n+1}}$ that $\alpha$ starts from and ends at respectively contributes a factor of $\lambda(s)$ as follows.

(s1) If $s$ is positive at the intersection $p$ of $\alpha$ and $\Delta_{i_{1}}$, then there is an ideal $\operatorname{arc} \alpha_{+}$in the state which is isotopic to $e_{r}$ as in $(s 1)$ of Figure 12. In this case $\Delta_{i_{1}}$ contributes a factor $\lambda_{r}$ to $\lambda(s)$.

(s2) If $s$ is negative at $p$, then there is an ideal arc $\alpha_{-}$in the state which is isotopic to $e_{l}$ as in $(s 2)$ of Figure 12. In this case $\Delta_{i_{1}}$ contributes a factor $\lambda_{l}$ to $\lambda(s)$.

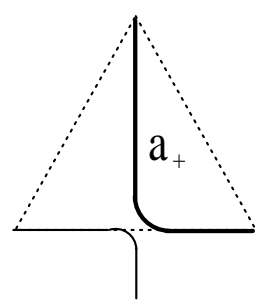

(s1)

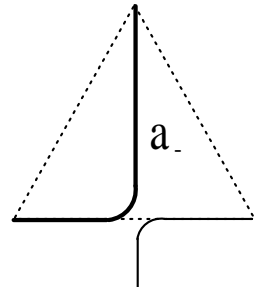

$(\mathrm{s} 2)$

Figure 12 
(t1) If $s$ is positive at the intersection $q$ of $\alpha$ and $\Delta_{i_{n+1}}$, then $\Delta_{i_{n+1}}$ contributes a factor $\lambda_{r}$ to $\lambda(s)$.

(t2) If $s$ is negative at $q$, then $\Delta_{i_{n+1}}$ contributes a factor $\lambda_{l}$ to $\lambda(s)$.

As such, each simultaneous resolution $s$ can be seen to correspond to a summand in the product $M_{1} \cdots M_{n+1}$ and the lambda lengths $\lambda(s)$ is equal to the value of the corresponding summand in $M_{1} \cdots M_{n+1}$. Therefore, we have $\sum_{s \in \mathcal{S}} \lambda(s)=M_{1} \cdots M_{n+1}$. As a consequence,

$$
\lambda(\alpha)=\frac{M_{1} \cdots M_{n+1}}{\lambda_{j_{1}} \cdots \lambda_{j_{n}}} .
$$

q.e.d.

In the case of a loop, one should compare this formula with the expression of trace functions in terms of shear coordinates which can be found in [BW11, Lemma 3].

\section{Appendix A. Cosine and sine laws of twisted generalized triangles}

1) Type $(1,1,-1)$ :

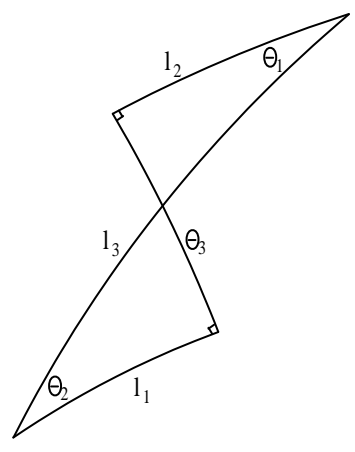

$$
\begin{array}{lrl}
\sinh l_{1}=\frac{-\cos \theta_{1}+\cos \theta_{2} \cosh \theta_{3}}{\sinh \theta_{2} \sin \theta_{3}} & \cos \theta_{1}=\frac{\sinh l_{1}+\sinh l_{2} \cosh l_{3}}{\cosh l_{2} \sinh l_{3}} \\
\sinh l_{2}=\frac{-\cos \theta_{2}+\cos \theta_{1} \cosh \theta_{3}}{\sin \theta_{1} \sinh \theta_{3}} & \cos \theta_{2}=\frac{\sinh l_{2}+\sinh l_{1} \cosh l_{3}}{\cosh l_{1} \sinh l_{3}} \\
\cosh l_{3}=\frac{\cosh \theta_{3}-\cos \theta_{1} \cos \theta_{2}}{\sin \theta_{1} \sin \theta_{2}} & \cosh \theta_{3}=\frac{\cosh l_{3}-\sinh l_{1} \sinh l_{2}}{\cosh l_{1} \cosh l_{2}} \\
\frac{\sin \theta_{1}}{\cosh l_{1}}=\frac{\sin \theta_{2}}{\cosh l_{2}}=\frac{\sinh \theta_{3}}{\sinh l_{3}} &
\end{array}
$$


2) Type $(1,0,-1)$ :

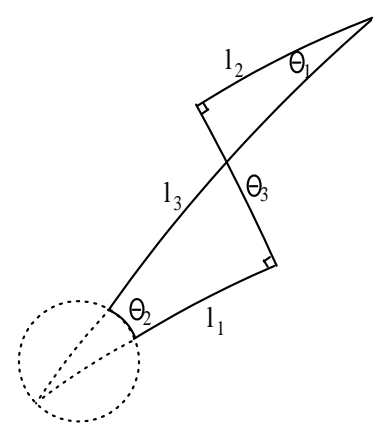

$$
\begin{array}{ll}
e^{l_{1}}=\frac{-\cos \theta_{1}+\cosh \theta_{3}}{\theta_{2} \sinh \theta_{3}} & \cos \theta_{1}=\frac{e^{l_{1}}+e^{l_{3}} \sinh l_{2}}{e^{l_{3}} \cosh l_{2}} \\
\sinh l_{2}=\frac{-1+\cos \theta_{1} \cosh \theta_{3}}{\sin \theta_{1} \sinh \theta_{3}} & \theta_{2}^{2}=\frac{-\sinh l_{2}+\sinh \left(l_{3}-l_{1}\right)}{\frac{e^{l_{1}+l_{3}}}{2}} \\
e^{l_{3}}=\frac{\cosh \theta_{3}-\cos \theta_{1}}{\theta_{2} \sin \theta_{1}} & \cosh \theta_{3}=\frac{e^{l_{3}}-e^{l_{1}} \sinh l_{2}}{e^{l_{1}} \cosh l_{2}} \\
\frac{\sin \theta_{1}}{e^{l_{1}}}=\frac{\theta_{2}}{\cosh l_{2}}=\frac{\sinh \theta_{3}}{e^{l_{3}}} &
\end{array}
$$

3) Type $(1,-1,-1)$ :

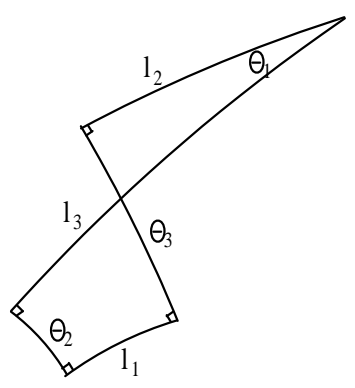

$$
\begin{array}{ll}
\cosh l_{1}=\frac{-\cos \theta_{1}+\cosh \theta_{2} \cosh \theta_{3}}{\sinh \theta_{2} \sinh \theta_{3}} & \cos \theta_{1}=\frac{\cosh l_{1}+\sinh l_{2} \sinh l_{3}}{\cosh l_{2} \cosh l_{3}} \\
\sinh l_{2}=\frac{-\cosh \theta_{2}+\cos \theta_{1} \cosh \theta_{3}}{\sin \theta_{1} \sinh \theta_{3}} & \cosh \theta_{2}=\frac{-\sinh l_{2}+\cosh l_{1} \sinh l_{3}}{\sinh l_{1} \cosh l_{3}} \\
\sinh l_{3}=\frac{\cosh \theta_{3}-\cos \theta_{1} \cosh \theta_{2}}{\sin \theta_{1} \sinh \theta_{2}} & \cosh \theta_{3}=\frac{\sinh l_{3}-\cosh l_{1} \sinh l_{2}}{\sinh l_{1} \cosh l_{2}} \\
\frac{\sin \theta_{1}}{\sinh l_{1}}=\frac{\sinh \theta_{2}}{\cosh l_{2}}=\frac{\sinh \theta_{3}}{\cosh l_{3}} &
\end{array}
$$


4) Type $(0,0,-1)$ :

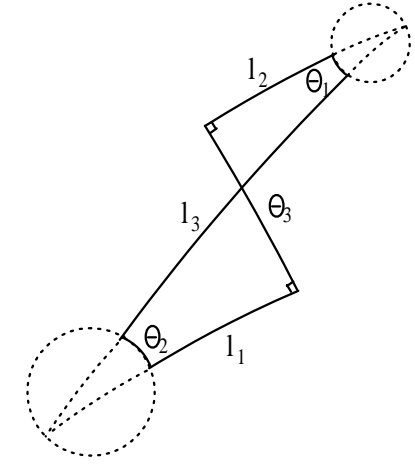

$$
\begin{array}{rlrl}
e^{l_{1}} & =\frac{-1+\cosh \theta_{3}}{\theta_{2} \sinh \theta_{3}} & \theta_{1}^{2}=\frac{-e^{l_{1}}+e^{l_{3}-l_{2}}}{e^{l_{2}+l_{3}}} \\
e^{l_{2}}=\frac{-1+\cosh \theta_{3}}{\theta_{1} \sinh \theta_{3}} & \theta_{2}^{2}=\frac{-e^{l_{2}}+e^{l_{3}-l_{1}}}{e^{l_{1}+l_{3}}} \\
e^{l_{3}}=\frac{\cosh \theta_{3}-1}{2 \theta_{1} \theta_{2}} & \cosh ^{2} \frac{\theta_{3}}{2}=e^{l_{3}-l_{1}-l_{2}} \\
\frac{\theta_{1}}{e^{l_{1}}}=\frac{\theta_{2}}{e^{l_{2}}}=\frac{\sinh \theta_{3}}{2 e^{l_{3}}} &
\end{array}
$$

5) Type $(0,-1,-1)$ :

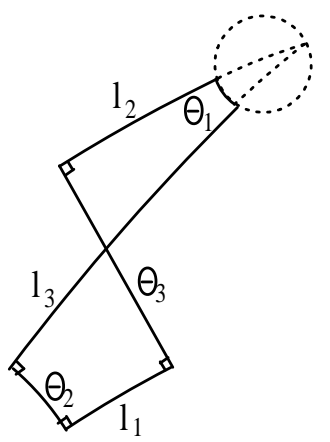

$$
\begin{array}{ll}
\cosh l_{1}=\frac{-1+\cosh \theta_{2} \cosh \theta_{3}}{\sinh \theta_{2} \sinh \theta_{3}} & \theta_{1}^{2}=\frac{-\cosh l_{1}+\cosh \left(l_{3}-l_{2}\right)}{\frac{e^{l_{2}+l_{3}}}{2}} \\
e^{l_{2}}=\frac{-\cosh \theta_{2}+\cosh \theta_{3}}{\theta_{1} \sinh \theta_{3}} & \cosh \theta_{2}=\frac{-e^{l_{2}}+e^{l_{3}} \cosh l_{1}}{e^{l_{3}} \sinh l_{1}} \\
e^{l_{3}}=\frac{\cosh \theta_{3}-\cosh \theta_{2}}{\theta_{1} \sinh \theta_{2}} & \cosh \theta_{3}=\frac{e^{l_{3}}-e^{l_{2}} \cosh l_{1}}{e^{l_{2}} \sinh l_{1}} \\
\frac{\theta_{1}}{\sinh l_{1}}=\frac{\sinh \theta_{2}}{e^{l_{2}}}=\frac{\sinh \theta_{3}}{e^{l_{3}}} &
\end{array}
$$


6) Type $(-1,-1,-1)$ :

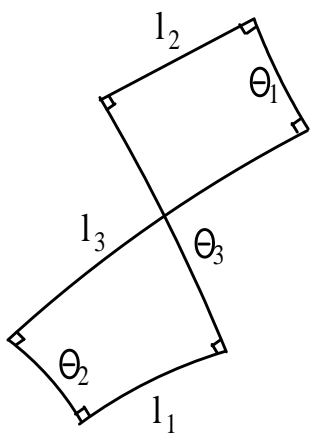

$$
\begin{aligned}
& \cosh l_{1}=\frac{-\cosh \theta_{1}+\cosh \theta_{2} \cosh \theta_{3}}{\sinh \theta_{2} \sinh \theta_{3}} \cosh \theta_{1}=\frac{-\cosh l_{1}+\cosh l_{2} \cosh l_{3}}{\sinh l_{2} \sinh l_{3}} \\
& \cosh l_{2}=\frac{-\cosh \theta_{2}+\cosh \theta_{1} \cosh \theta_{3}}{\sinh \theta_{1} \sinh \theta_{3}} \cosh \theta_{2}=\frac{-\cosh l_{2}+\cosh l_{1} \cosh l_{3}}{\sinh l_{1} \sinh l_{3}} \\
& \cosh l_{3}=\frac{\cosh \theta_{3}-\cosh \theta_{1} \cosh \theta_{2}}{\sinh \theta_{1} \sinh \theta_{2}} \quad \cosh \theta_{3}=\frac{\cosh l_{3}-\cosh l_{1} \cosh l_{2}}{\sinh l_{1} \sinh l_{2}} \\
& \frac{\sinh \theta_{1}}{\sinh l_{1}}=\frac{\sinh \theta_{2}}{\sinh l_{2}}=\frac{\sinh \theta_{3}}{\sinh l_{3}}
\end{aligned}
$$

\section{References}

[AU11] J.E. Andersen \& K. Ueno, Construction of the Reshetikhin-Turaev TQFT from conformal field theory. Preprint, arXiv:1110.5027.

[BZ05] A. Berenstein \& A. Zelevinsky, Quantum cluster algebras, Adv. Math. 195 (2005), no. 2, 405-455, MR 2146350, Zbl 1124.20028.

[BHMV95] C. Blanchet, N. Habegger, G. Masbaum \& P. Vogel, Topological quantum field theories derived from the Kauffman bracket. Topology 34 (1995), no. 4, 883-927, MR 1362791, Zbl 0887.57009.

[Bo96] F. Bonahon, Shearing hyperbolic surfaces, bending pleated surfaces and Thurston's symplectic form. Ann. Fac. Sci. Toulouse Math. (6) 5 (1996), 233-297, MR 1413855, Zbl 0880.57005.

[BW10] F. Bonahon, Kauffman brackets, character varieties and triangulations of surfaces. Topology and geometry in dimension three, 179-194, Contemp. Math., 560, Amer. Math. Soc., Providence, RI, 2011. MR 2866931.

[BW11] F. Bonahon \& H. Wong, Quantum traces for representations of surface groups in $S L_{2}(\mathbb{C})$, Geom. Topol. 15 (2011), no. 3, 1569-1615, MR 2851072, Zbl 1227.57003.

[Bu97] D. Bullock, Rings of $S L_{2}(\mathbf{C})$-characters and the Kauffman bracket skein module. Comment. Math. Helv. 72 (1997) no. 4, 521-542, MR 1600138, Zbl 0907.57010.

[BFK98] D. Bullock, C. Frohman \& J. Kania-Bartoszyńska, Topological interpretations of lattice gauge field theory. Comm. Math. Phys. 198 (1998), no. 1, 47-81, MR 1657365, Zbl 0916.53046. 
[BFK99] D. Bullock, C. Frohman \& J. Kania-Bartoszyńska, Understanding the Kauffman bracket skein module. J. Knot Theory Ramifications 8 (1999), no. 3, 265-277, MR 1691437, Zbl 0932.57015.

[CF99] L. O. Chekhov, \& V. V. Fock, Quantum Teichmüller spaces. Theor. Math. Phys. 120 (1999), 1245-1259, MR 1737362.

[CM09] L. Charles \& J. Marché, Multicurves and regular functions on the representation variety of a surface in $S U(2)$. Preprint, arXiv:0901.3064.

[DP11] G. Dupont \& F. Palesi, Quasi-cluster algebras from non-orientable surfaces. Preprint, arXiv:1105.1560.

[FG06] V. V. Fock \& A. Goncharov, Moduli spaces of local systems and higher Teichmüller theory. Publ. Math. Inst. Hautes Études Sci. No. 103 (2006), 1-211, MR 2233852, Zbl 1099.14025.

[FWW02] M.H. Freedman, K. Walker, Z. Wang, Quantum SU(2) faithfully detects mapping class groups modulo center. Geom. Topol. 6 (2002), 523-539, MR 1943758, Zbl 1037.57024.

[Go86] W. M. Goldman, Invariant functions on Lie groups and Hamiltonian flows of surface group representations. Invent. Math. 85 (1986), no. 2, 263-302, MR 0846929, Zbl 0619.58021.

[GL09] R. Guo \& F. Luo, Rigidity of polyhedral surfaces. II. Geom. Topol. 13 (2009), 1265-1312, MR 2496046, Zbl 1160.52012.

[Hi76] M.W. Hirsch, Differential Topology. Graduate texts in mathematics 33, BerlinHeidelberg-New York: Springer 1976, MR 1336822, Zbl 0356.57001.

[Ka98] R. Kashaev, Quantization of Teichmüller spaces and the quantum dilogarithm. Lett. Math. Phys. 43 (1998), 105-115, MR 1607296, Zbl 0897.57014.

[Kas95] C. Kassel, Quantum groups. Graduate Texts in Mathematics, 155. SpringerVerlag, New York, 1995. MR 1321145, Zbl 0808.17003.

[Kau90] L. H. Kauffman, An invariant of regular isotopy. Trans. Amer. Math. Soc. 318 (1990), no. 2, 417-471, MR 0958895, Zbl 0763.57004.

[KS98] L. I. Korogodski \& Y.S. Soibelman, Algebras of functions on quantum groups. Part I. Mathematical Surveys and Monographs, 56. American Mathematical Society, Providence, RI, 1998. MR 1614943, Zbl 0923.17017.

[Mo09] G. Mondello, Triangulated Riemann surfaces with boundary and the WeilPetersson Poisson structure. J. Differential Geom. 81 (2009), 391-436, MR 2472178, Zbl 1165.53026.

[MW11] G. Musiker \& L. Williams, Matrix formulae and skein relations for cluster algebras from surfaces. Preprint, arXiv:1108.3382.

[Pe87] R.C. Penner, The decorated Teichmüller space of punctured surfaces. Comm. Math. Phys. 113 (1987), 299-339, MR 0919235, Zbl 0642.32012.

[Pe92] R. C. Penner, Weil-Petersson volumes. J. Differential Geom. 35 (1992), no. 3, 559608, MR 1163449, Zbl 0768.32016.

[Pr91] J. H. Przytycki, Skein modules of 3-manifolds. Bull. Polish Acad. Sci 39 (1991) 91-100, MR 1194712.

[PS00] J.H. Przytycki \& A. S. Sikora, On skein algebras and $S L_{2}(\mathbf{C})$-character varieties. Topology 39 (2000), no. 1, 115-148, MR 1710996, Zbl 0958.57011.

[Tu88] V. G. Turaev, The Conway and Kauffman modules of a solid tours. (Russian). Zap. Nauchn. Sem. Leningrad. Otdel. Mat. Inst. Steklov. (LOMI) 167 (1988), Issled. 
Topol. 6, 79-89, 190; translation in J. Soviet Math. 52 (1990), no. 1, 2799-2805. MR 0964255.

[Tu91] V.G. Turaev, Skein quantization of Poisson algebras of loops on surfaces. Ann. Sci. École Norm. Sup. (4) 24 (1991), no. 6, 635-704, MR 1142906, Zbl 0758.57011.

[Wh37] H. Whitney, On regular closed curves in the plane. Comp. Math. 4 (1937), 276284, MR 1556973, Zbl 0016.13804.

[Wo83] S. Wolpert, On the symplectic geometry of deformations of a hyperbolic surface. Ann. of Math. (2) 117 (1983), no.2, 207-234, MR 0690844, Zbl 0518.30040.

DEPARTMENT OF MATHEMATICS RUTGERS UNIVERSITY

NEW BRUNSWICK, NJ 08854, USA

E-mail address: juroger@math.rutgers.edu

DEPARTMENT OF MATHEMATICS RUTGERS UNIVERSITY

NEW BRUNSWICK, NJ 08854, USA

E-mail address: tianyang@math.rutgers.edu 